\title{
Optimization of an Aeroservoelastic Wing with Distributed Multiple Control Surfaces
}

\author{
Bret K. Stanford ${ }^{1}$ \\ NASA Langley Research Center, Hampton, VA, 23681
}

\begin{abstract}
This paper considers the aeroelastic optimization of a subsonic transport wingbox under a variety of static and dynamic aeroelastic constraints. Three types of design variables are utilized: structural variables (skin thickness, stiffener details), the quasi-steady deflection scheduling of a series of control surfaces distributed along the trailing edge for maneuver load alleviation and trim attainment, and the design details of an LQR controller, which commands oscillatory hinge moments into those same control surfaces. Optimization problems are solved where a closed loop flutter constraint is forced to satisfy the required flight margin, and mass reduction benefits are realized by relaxing the open loop flutter requirements.
\end{abstract}

\section{Introduction}

A eroelastic tailoring of wing structures has typically consisted of optimizing the thickness distribution of the various wing box components (skins, spars, etc.), or optimizing the stacking sequence, in the case of composite structures [1]. Design metrics may include static aeroelastic trim stresses, panel buckling, aeroelastic stability (divergence, flutter), gust response, maneuver/trim drag, etc. In addition to structural sizing variables, one may also consider distributed multiple control surfaces (DMCS) along the trailing edge of the wing (and, potentially, the leading edge). Optimizing the static and dynamic deflections of these control surfaces would change the apparent stiffness and mass of the wing structure, rather than the true quantities, but these divisions are blurred in any case, due to the coupled nature of an aeroelastic system. Distributed control surfaces may be equally adept at altering load paths and dynamic behavior of a wing structure for improved performance in terms of any of the design metrics mentioned above. Furthermore, simultaneously designing the wing structure and the actuator deflections will allow an optimizer to take advantage of the synergies between the two types of design variables.

Distributed multiple control surfaces may be used in two distinct ways [2]. First, their deflection patterns may be scheduled in a quasi-steady manner for maneuver load alleviation. A separate deflection pattern may be optimized for each trim load case, where the distributed control surfaces would simultaneously alleviate the loads into the wing structure and help maintain aerodynamic trim. This may be done by solving an over-determined trim problem, where flap deflection scheduling is optimized such that trim is maintained (posed as a constraint) and some objective of interest is minimized: root bending moment [3], deformations at given finite element nodes [4], drag, etc. This typically is posed as a nested optimization solution, where structural design variables are applied to the over-determined trim solution. Alternatively, structural design variables may be optimized concurrently with the control surface scheduling [5], either iteratively or simultaneously. This latter scenario is of interest for this work.

Secondly, the control surfaces may be used for active flutter mitigation or gust rejection, where each flap dynamically oscillates about the steady-state position found with the methods in the previous paragraph (superposition). This introduces a $3^{\text {rd }}$ type of design variable into the aeroelastic optimization (in addition to the structural variables and steady flap deflection variables): the details of the controller. Though aeroservoelastic optimization may be accomplished through a variety of means [2], one convenient technique is to use a linear quadratic controller, and optimize the details of the weighting matrices in the quadratic performance index (controller cost function). This is demonstrated in Refs. [6], [7], and [8]. Furthermore, the performance index itself may be used as an objective function or constraint [9][10][11] during the optimization process. Papers that study the use of multiple and/or distributed control surfaces for aeroservoelastic control (though not for optimization) can be found in Refs. [12], [13], and [14].

\footnotetext{
${ }^{1}$ Research Aerospace Engineer, Aeroelasticity Branch, bret.k.stanford@nasa.gov, AIAA Member.
} 
Aeroservoelasticity in general is concerned with flutter, gust alleviation, ride quality, etc. Of these dynamic performance metrics, the current paper only considers flutter, in an effort to understand the structural weight savings that may be gained when an open loop flutter margin constraint is relaxed, and closed loop control is instead used to impose the required safety margin. This work is conducted on the un-deflected Common Research Model (uCRM) [15], a jig shape of the flying wing model developed in Ref. [16]. The uCRM is a generic subsonic transport configuration with a standard rib-spar wingbox, and has been outfitted for this work with a series of control surfaces distributed along the trailing edge.

As noted above, three types of design variables are considered here, which in this paper are introduced into the aeroelastic optimization problem in stages. First (Section IV), only structural design variables (skin thickness and stiffener geometry for the skins, ribs, and spars) are used to minimize the mass of the wingbox, under static aeroelastic constraints only. Next (Section V), the quasi-steady deflections of each control surface (DMCS), at each maneuver load case, are added to the vector of design variables, and the optimization problem is re-solved. The impact (on the minimum attainable mass) of including an open loop flutter constraint is then assessed (Section VI). Finally (Section VII), controller design variables are added to the design variable vector, and a closed loop flutter constraint is imposed, along with the open loop constraint. Trade-offs (Pareto fronts) between optimal wing mass, open loop flutter margin, and control cost (performance index) are all presented.

\section{The Common Research Model}

All of the work in this paper is conducted on the conceptual Common Research Model (CRM). The model presented in Ref. [16] is a $1 \mathrm{~g}$ flying shape suitable for rigid aerodynamic analysis. An un-deflected jig shape version (uCRM) developed in Ref. [15] is suitable for aeroelastic analysis, and is used here. This transonic transport configuration has a wing span of $58.7 \mathrm{~m}$, a mean aerodynamic chord of $7.0 \mathrm{~m}$, an aspect ratio of 9 , a taper ratio of 0.275 , a sweep angle of $35^{\circ}$, and a cruise Mach number of 0.85 .

The topology of the wingbox developed in Ref. [15] is also used here, seen in Figure 1. This structure consists of an upper skin, a lower skin, a leading edge spar (located at 10\% chord at the root and $35 \%$ at the tip), a trailing edge spar (60\% at both the root and the tip), and 43 ribs oriented perpendicular to the leading edge. All shell members (ribs, spars, skins) are outfitted with blade stiffeners, where the pitch is equal to $0.33 \mathrm{~m}$ throughout. Runout stiffeners are utilized down the span: 18 stiffeners are found for panels and ribs at the root, but only 3 at the tip. The stiffeners are not modeled explicitly, but instead smeared into the shell stiffness properties. The entire wing structure is constructed of aluminum ( $E=70 \mathrm{GPa}, v=0.3, \rho=2780 \mathrm{~kg} / \mathrm{m}^{3}, \sigma_{Y}=330 \mathrm{MPa}$ ), and discretized into 16,000 triangular finite elements.

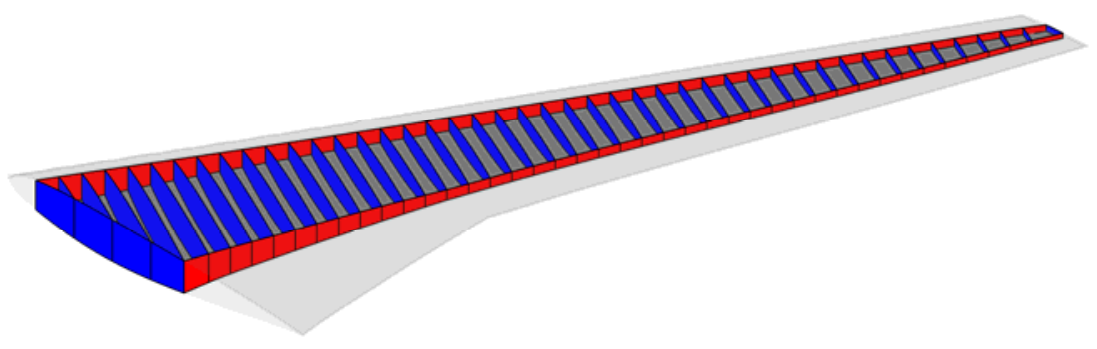

Figure 1. uCRM wingbox topology and outer mold line, taken from Ref. [15].

Twenty simple flap-like control surfaces are distributed along the trailing edge of the wing from root to chord, as shown in Figure 2. A hinge spring attached to each control surface is connected back to the trailing spar via interpolation elements. The mass of each DMCS is assumed proportional to the volume enclosed within the surface wedge, and the stiffness of each hinge spring is sized such that all of the control surfaces have the same natural vibration frequency. The structure within the leading edge of the wing is not explicitly modeled, though an inertial effect is captured with a series of lumped masses attached to the wing box via interpolation elements. These masses, along with similar representations for the engine and the fuel, are also shown in Figure 2. In addition to the inertial loads from the lumped masses, a thrust force is applied to the central engine node.

Aerodynamic paneling for the wing, horizontal tail, vertical tail, fuselage, and engine (the latter two represented as cruciforms) is shown in Figure 3, with a total of 4,600 panels. For static aeroelastic trim analysis, the entire vehicle representation of Figure 3 is utilized, and trailing edge control surface hinge springs are modeled as rigid. In this case, commanded quasi-steady flap deflections (relative to the wing) are exactly obtained. For dynamic flutter 
analysis, only the wing panels are utilized. Furthermore, the control surfaces are now allowed to vibrate freely, and commanded flap deflections may be resisted by aerodynamic and inertial hinge moments.

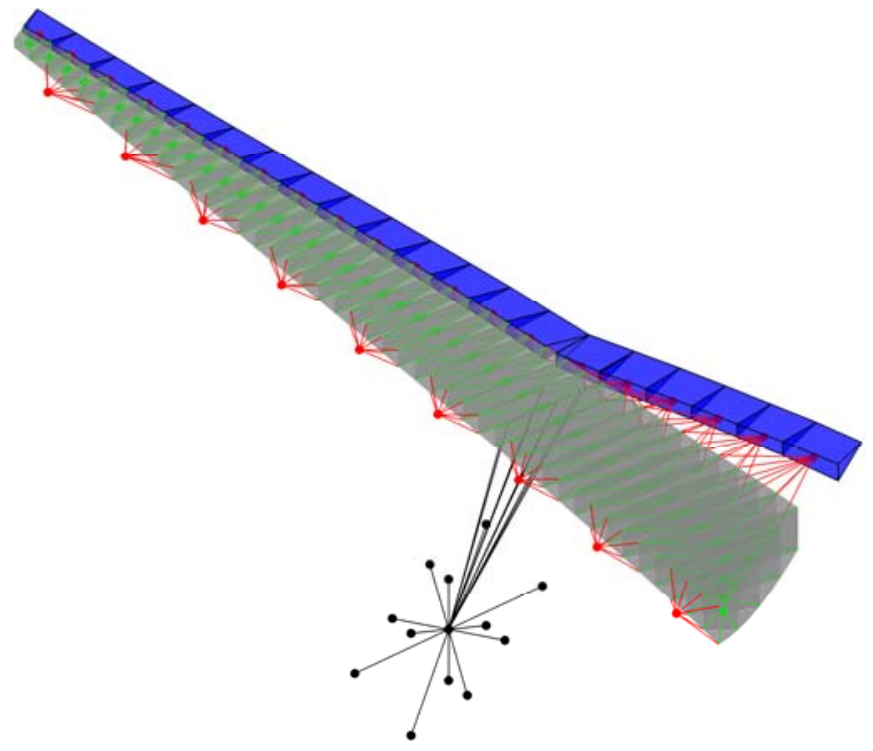

Figure 2. Control surfaces attached to the uCRM model, and lumped mass representation of engine and fuel loading.

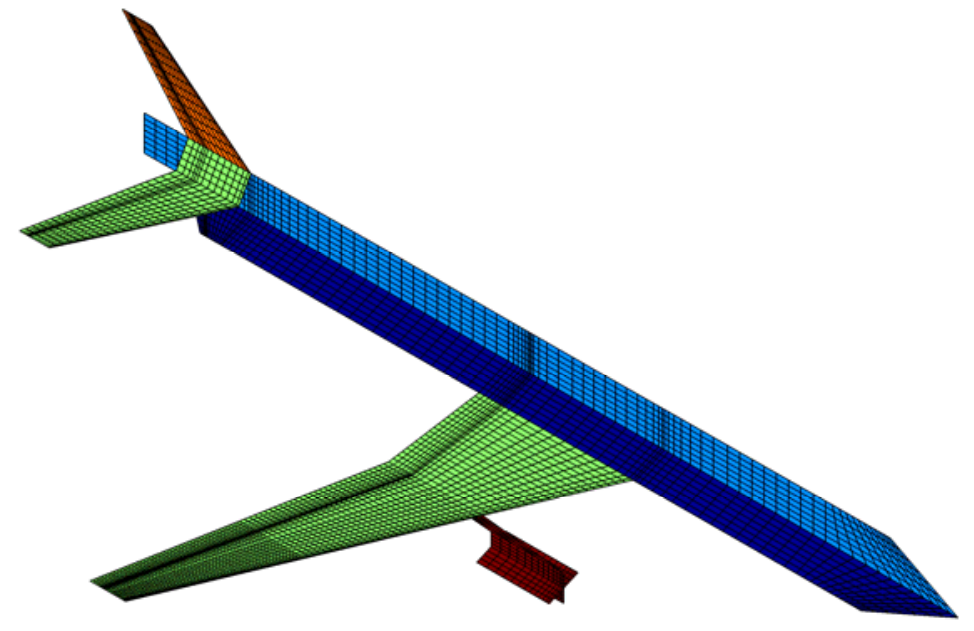

Figure 3. Aerodynamic paneling of the uCRM.

\section{Static Aeroelastic Modeling}

\section{A. Trim Loads}

The shell finite elements used to model the wing structure are defined by a combination of linear strain triangles (LST) and discrete Kirchhoff triangles (DKT) [17]. For static airloads, a linear vortex lattice method [18] is used to model the aerodynamic lifting surfaces. A finite plate spline (FPS) method [19] is used to transfer downwash and pressures between the aerodynamic and structural/control surface modules. Only information pertaining to the wing is transferred in this way: the remaining aerodynamic surfaces are not explicitly tied to any structure.

The wing box structure is sized across two different types of static maneuvers. The first type is a longitudinal maneuver (pull-up, push-over), where the system is trimmed via the angle of attack $\alpha$ and the elevator deflection $\delta$. The trimmed values of these two quantities are automatically found by augmenting the aero-structural coupling equations. The final equation for a longitudinal maneuver is written as: 


$$
\left[\begin{array}{cccc}
\boldsymbol{K} & -q \cdot \boldsymbol{Q} & \mathbf{0} & \mathbf{0} \\
-\boldsymbol{P} & \boldsymbol{D}_{s} & -\boldsymbol{L}_{\alpha} & -\boldsymbol{L}_{\delta} \\
\mathbf{0} & q \cdot \boldsymbol{S}_{L}^{T} & 0 & 0 \\
\mathbf{0} & q \cdot \boldsymbol{S}_{m}^{T} & 0 & 0
\end{array}\right] \cdot\left\{\begin{array}{c}
\boldsymbol{x}_{s} \\
\boldsymbol{C}_{p} \\
\alpha \\
\delta
\end{array}\right\}=\left\{\begin{array}{c}
N \cdot \boldsymbol{F}_{\text {grav }}+\boldsymbol{F}_{\text {thrust }} \\
\boldsymbol{z}_{j i g} \\
N \cdot W \\
0
\end{array}\right\}+\left\{\begin{array}{c}
\mathbf{0} \\
\boldsymbol{L}_{\gamma} \cdot \boldsymbol{\gamma}_{s} \\
0 \\
0
\end{array}\right\}
$$

The first row of Eq. 1 is the finite element analysis: $\boldsymbol{K}$ is the stiffness matrix, and the solution vector $\boldsymbol{x}_{\boldsymbol{s}}$ has six degrees of freedom per node (three displacements and three rotations). The $s$ subscript indicates a "symmetric" term, to differentiate from the anti-symmetric terms below. Forcing functions include self-weight inertial loading $\boldsymbol{F}_{\text {grav }}$ (scaled by the maneuver load factor $N$, and accounting for both the weight of the wing structure and the lumped masses in Figure 2), thrust loading $\boldsymbol{F}_{\text {thrust }}$ from the engine, and aerodynamic forces. Aerodynamic forces are written as $q \cdot \boldsymbol{Q} \cdot \boldsymbol{C}_{p}$, where $\boldsymbol{C}_{p}$ is a vector of differential pressure coefficients acting on each panel of the vehicle, $\boldsymbol{Q}$ is an interpolation function derived from FPS, and $q$ is the dynamic pressure.

The second row of Eq. 1 is the aerodynamic analysis, where $\boldsymbol{D}_{s}$ is the matrix of aerodynamic influence coefficients (AIC) and the subscript indicates a symmetric aerodynamic condition about the centerline of the airplane in Figure 3. This equation is driven by several terms: downwash due to angle of attack $\boldsymbol{L}_{\alpha} \cdot \alpha$ (where $\boldsymbol{L}_{\alpha}$ is a linear operator that converts the scalar angle of attack into a downwash at each panel), elevator deflection $\boldsymbol{L}_{\delta} \cdot \delta$, built in camber/twist of the wing and tail jig shapes $\boldsymbol{z}_{j i g}$, and downwash induced by structural wing deformation. This latter term is written as $\boldsymbol{P} \cdot \boldsymbol{x}_{s}$, where $\boldsymbol{P}$ is a second interpolation function, also derived from FPS-based methods. A final downwash term is needed if distributed control surfaces are used along the trailing edge during the maneuver. The deflection of each control surface is grouped into the vector $\boldsymbol{\gamma}_{s}$, and $\boldsymbol{L}_{\gamma}$ is a matrix that converts these deflections into the appropriate downwash at each panel.

These deflections $\gamma_{s}$ are known, specified quantities during the solution of Eq. 1: $\alpha$ and $\delta$ are found that trim the system in the presence of these and other terms. Trim equations are written in the $3^{\text {rd }}$ and $4^{\text {th }}$ rows of Eq. 1: $q \cdot \boldsymbol{S}_{L}$ and $q \cdot \boldsymbol{S}_{m}$ convert the differential pressure vector $\boldsymbol{C}_{p}$ into a total aerodynamic lift and aerodynamic pitching moment (about the aircraft center of gravity). Lift must offset the total weight of the vehicle $(N \cdot W)$, and the net pitching moment must be zero.

A second type of static maneuver considered here is a rolling trim analysis (Eq. 2), where the deflection $\beta$ of an outboard wing aileron is found such that a constant specified non-dimensional roll rate $p \cdot L / U$ is maintained, with no rolling acceleration. In this analysis, $p$ is the dimensional roll rate, $L$ is the semi-span, and $U$ is the flight speed. The "aileron" is formed by grouping the 4 control surfaces placed between $70 \%$ and $90 \%$ of the semi-span in Figure 2. The system is simultaneously trimmed longitudinally for steady level flight $(N=1)$ with the angle of attack $\alpha$. The rolling analysis requires an anti-symmetric condition about the centerline of the airplane; the level flight anlaysis uses a symmetric condition. Owing to the linear nature of the methods used here, structural deformations and aerodynamic pressures for the two conditions can be solved separately, and then added together to obtain the total aeroelastic state. The final equation for this maneuver (rolling at level flight) is written as:

$$
\left[\begin{array}{cccccc}
\boldsymbol{K} & \mathbf{0} & -q \cdot \boldsymbol{Q} & \mathbf{0} & \mathbf{0} & \mathbf{0} \\
\mathbf{0} & \boldsymbol{K} & \mathbf{0} & -q \cdot \boldsymbol{Q} & \mathbf{0} & \mathbf{0} \\
-\boldsymbol{P} & \mathbf{0} & \boldsymbol{D}_{s} & \mathbf{0} & -\boldsymbol{L}_{\alpha} & \mathbf{0} \\
\mathbf{0} & -\boldsymbol{P} & \mathbf{0} & \boldsymbol{D}_{a} & \mathbf{0} & -\boldsymbol{L}_{\beta} \\
\mathbf{0} & \mathbf{0} & q \cdot \boldsymbol{S}_{L}^{T} & \mathbf{0} & 0 & 0 \\
\mathbf{0} & \mathbf{0} & \mathbf{0} & q \cdot \boldsymbol{S}_{p}^{T} & 0 & 0
\end{array}\right] \cdot\left\{\begin{array}{c}
\boldsymbol{x}_{s} \\
\boldsymbol{x}_{a} \\
\boldsymbol{C}_{p, s} \\
\boldsymbol{C}_{p, a} \\
\alpha \\
\beta
\end{array}\right\}=\left\{\begin{array}{c}
N \cdot \boldsymbol{F}_{\text {grav }}+\boldsymbol{F}_{\text {thrust }} \\
\mathbf{0} \\
\boldsymbol{z}_{j i g} \\
\left(\frac{p \cdot L}{U}\right) \cdot \boldsymbol{L}_{p} \\
W \\
0
\end{array}\right\}+\left\{\begin{array}{c}
\mathbf{0} \\
\mathbf{0} \\
\boldsymbol{L}_{\gamma} \cdot \boldsymbol{\gamma}_{s} \\
\boldsymbol{L}_{\gamma} \cdot \boldsymbol{\gamma}_{a} \\
0 \\
0
\end{array}\right\}
$$

The $a$ subscripts indicates an "anti-symmetric" term, $q \cdot \boldsymbol{S}_{p}$ converts the aerodynamic pressures into a rolling moment about the centerline (which is ultimately set to zero for a constant roll rate), and $\boldsymbol{L}_{p}$ converts the rolling motion into a downwash term. It is also seen that distributed control surfaces along the trailing edge, if used, are separated into symmetric and anti-symmetric scheduling. Trailing edge control surfaces used to define the $\beta$ rotation in the trim module are excluded from the $\gamma_{a}$ terms.

\section{B. Stresses}

Having solved the static trim Eqs. 1 and 2, stresses and strains can be computed for each static load case, and a knock-up (safety) factor of $30 \%$ is applied to each elemental stress value. The von Mises failure function is computed for each finite element. The spar, rib, and skin structures are then divided into patches (seen in Figure 1, with a total of 217 patches), and the Kreisselmeier-Steinhauser (KS) function [20] is used to compress all of the 
elemental failure function values within a given patch into a single metric. If all of the stress values within a patch are within their failure envelope, the KS function for that patch will be less than one.

\section{Panel Buckling}

After the stress analysis, buckling analyses are run for each stiffened panel in the upper and lower skins. Following Refs. [21] and [22], each buckling analysis is conducted with a Rayleigh-Ritz method (assumed buckling modes). Both global buckling of a stiffened panel (bordered by ribs and spars) and local buckling in between each stiffener is computed, where simply supported boundary conditions are used for both scenarios. The buckling equation is given as:

$$
\left(\boldsymbol{K}_{s}+\mu_{n} \cdot \widetilde{\boldsymbol{K}}\right) \cdot \boldsymbol{v}_{n}=\mathbf{0}
$$

where $\boldsymbol{v}_{n}$ is the eigenvector (vector of modal amplitudes) associated with the $n^{\text {th }}$ eigenvalue $\mu_{n}$. The panel stiffness matrix $\widetilde{\boldsymbol{K}}$ is computed based upon the stiffness properties defined at the wing level. Smeared stiffness properties are necessarily used for global panel buckling, whereas un-smeared shell properties are used for local inter-stringer buckling. The panel's geometric stiffness matrix $\boldsymbol{K}_{s}$ is assembled with element stresses computed from Eqs. 1 or 2 .

An eigenvalue $\mu_{n}$ greater than one indicates that, for the trimmed aeroelastic state, the panel has buckled. Each buckling computation for a given stiffened panel is compressed into a single value using a KreisselmeierSteinhauser function. For a panel with 3 stringers (such as found at the wing tip), 4 local buckling computations are performed, along with 1 global buckling computation. Since three modes $(n=3)$ are computed for each eigenproblem, a total of 15 eigenvalues are compressed into a single KS function for this example. The total number of stiffened panels in Figure 1 along the upper and lower skins is 88, which is also equal to the number of KS buckling values.

\section{Design Variables and Analytical Sensitivities}

Structural design variables for static aeroelastic optimization include shell thickness, stiffener thickness, and stiffener height, where simple blade stiffeners are used. These variables will have an impact on the wing and panel stiffness matrices $\boldsymbol{K}, \widetilde{\boldsymbol{K}}$, and $\boldsymbol{K}_{s}$, the inertial loading $\boldsymbol{F}_{\text {grav }}$, the vehicle weight $W$, and the term $\boldsymbol{S}_{m}$ (which is dependent upon the center of gravity). In the event that the scheduling of distributed control surface deflections are used during the optimization, the relevant design variables are $\boldsymbol{\gamma}_{s}$ and $\boldsymbol{\gamma}_{a}$. One set of symmetric deflections $\boldsymbol{\gamma}_{s}$ is designed for each load case (Eq. 1 or 2), and a set of anti-symmetric deflections $\left(\gamma_{a}\right)$ are further included for each rolling load case (Eq. 2).

Derivatives of the static aeroelastic response (stress and buckling aggregation parameters for each load case) with respect to all design variables are computed with the adjoint method.

\section{Static Aeroelastic Optimization with Structural Design Variables}

The optimal distribution of patch-based skin thickness, stiffener thickness, and stiffener height variables (using the rib, spar, and skin design patches in Figure 1) are found that minimizes the wing mass (based on the volume of the finite element model) subject to aeroelastic strength and buckling constraints:

$$
\begin{aligned}
& \underset{\boldsymbol{q}}{\min } \text { mass } \\
& \text { s.t. } \begin{cases}\mathbf{0} \leq \boldsymbol{q} \leq \mathbf{1} & \\
K S_{\sigma, i} \leq 1 & i=1, \ldots,\left(N_{\sigma} \cdot N_{L}\right) \\
K S_{\mu, i} \leq 1 & i=1, \ldots,\left(N_{\mu} \cdot N_{L}\right)\end{cases}
\end{aligned}
$$

where $\boldsymbol{q}$ are the design variables that have been appropriately normalized between 0 and 1 . Dimensionally, shell thicknesses are allowed to range between $3 \mathrm{~mm}$ and $30 \mathrm{~mm}$, stiffener thicknesses between $3 \mathrm{~mm}$ and $30 \mathrm{~mm}$, and stiffener heights between $30 \mathrm{~mm}$ and $150 \mathrm{~mm}$.

$N_{L}$ is the number of static load cases, $K S_{\sigma}$ are the stress aggregation parameters ( $N_{\sigma}$ per load case), $K S_{\mu}$ are the buckling aggregation parameters ( $N_{\mu}$ per load case). Three static load cases are considered for all optimization problems in this paper. Each has a Mach number of 0.85 and a dynamic pressure of $13.8 \mathrm{kPa}$. The first two load cases are longitudinal maneuvers (Eq. 1), with load factors of 2.5 and -1 , respectively. The third load case is 
dictated by Eq. 2, where the desired non-dimensional roll rate is 0.065 . All cases are run with half-fuel, and the distributed control surfaces along the trailing edge (except the aileron deflection $\beta$ used for lateral trim) are ignored: $\boldsymbol{\gamma}_{S}=\boldsymbol{\gamma}_{a}=\mathbf{0}$. Quasi-steady control surface deflection design variables will be considered in section V.

The design variables $\boldsymbol{q}$ are passed through a linearly-decaying cone-shape filter [23] prior to conversion into structural quantities, in order to prevent the difference in stiffness between adjacent patches from being too large. All optimization problems are solved with the Globally-Convergent Method of Moving Asymptotes [24]. The optimal result is shown in Figure 4, in terms of the shell thickness and smeared stiffener height of each design patch. The smeared stiffener thickness of each patch resides at the prescribed lower bound of $3 \mathrm{~mm}$, and is not shown. The optimizer places the majority of the material in the upper and lower skins, with thickness and stiffener height tapered towards the lower bound at the wing tip, due to the drop in bending moment. Much larger stiffeners are utilized in the upper skins as compared to the lower skins, which is a buckling-driven result (discussed below). Rib design variables are all pushed to the lower bound, as are many of the spar variables, with the exception of the thick trailing edge root spar. Crushing loads are not included here, which may drive the optimizer to strengthen the rib members.

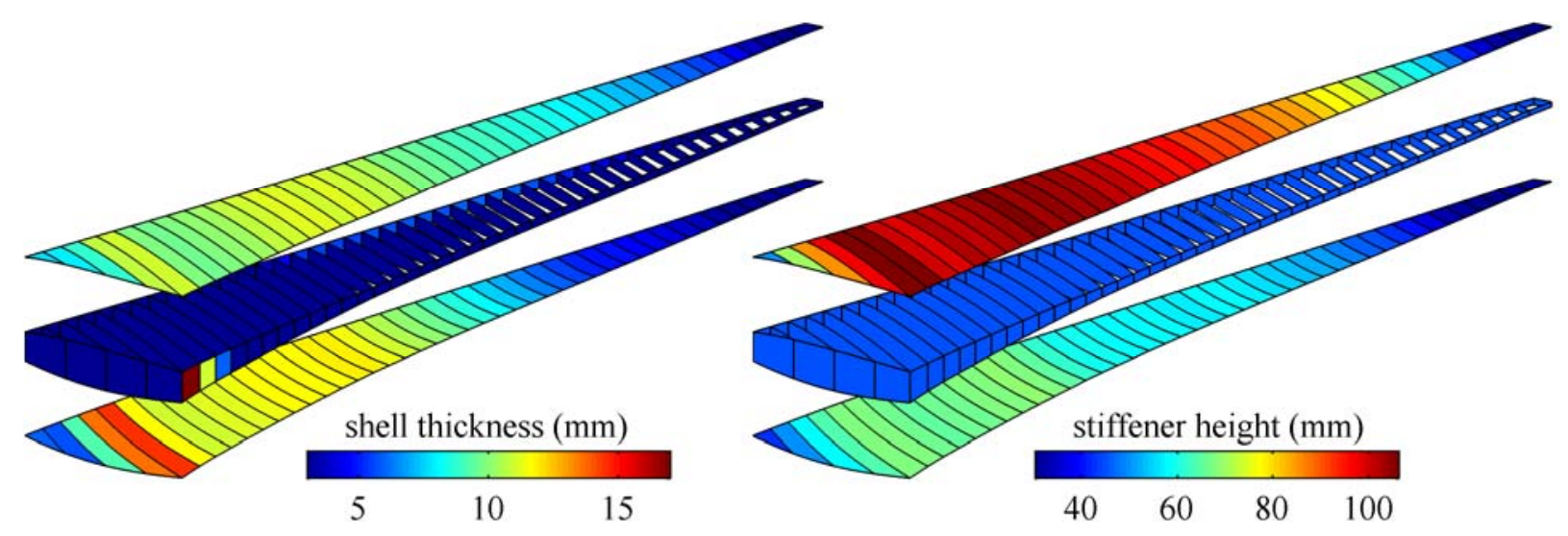

Figure 4. Optimal structural design variables for the static aeroelastic optimization problem.

Structural deflections for each load case are given in Figure 5, element-level von Mises failure functions in Figure 6, and KS buckling parameters in Figure 7. It is noted that the data in Figure 6 are not explicitly provided to the optimizer, but first compressed into $K S_{\sigma}$ functions for each design segment. The buckling data in Figure 7 is $K S_{\mu}$, and therefore is provided to the optimizer in the form shown. As discussed above, each $K S_{\mu}$ is an aggregation of each global and local (inter-stringer) buckling eigenvalue for a given stiffened panel. For both stress data and buckling data, a value greater than one would be an infeasible aeroelastic constraint, and is not seen in either plot.

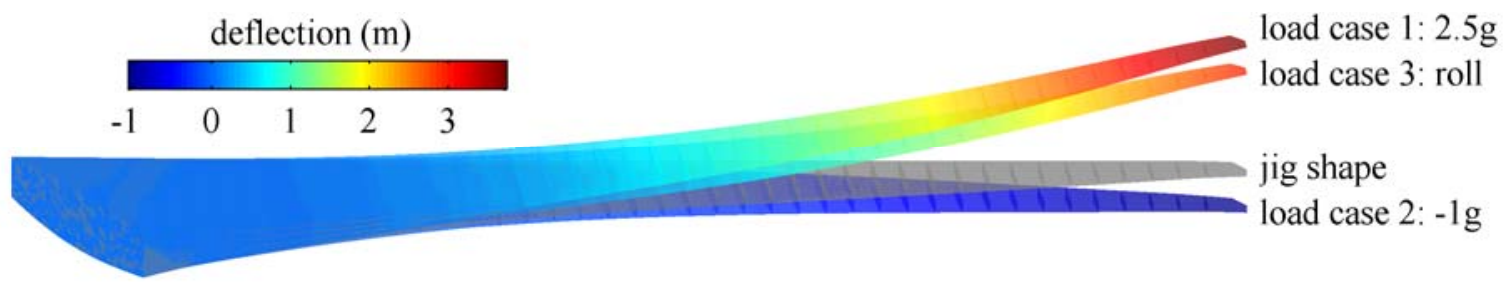

Figure 5. Structural deflections of the optimal design in Figure 4 across each load case.

Wing deflections in Figure 5 follow the expected pattern, though the rolling load case 3 has deflections nearly as large as the pull-up maneuver (2.5g). Furthermore, this trimmed rolling maneuver does not qualitatively show the torsion-dominated response that may be expected. The stress distribution (Figure 6) is markedly different however, with peak skin stresses shifted further outboard than seen for the longitudinal maneuvers. Large portions of the outboard spars are stressed for this rolling maneuver as well, whereas for the longitudinal maneuvers, high spar stresses are confined to the engine attachment points. For load case 2 (-1g push over), these engine stresses are the critical values for the entire load case: stresses in the skins are relatively low. For load case 1, however, the majority of the upper and lower skin stresses are close to the allowable limit, which is a nearly fully-stressed design. Rib stresses are uniformly low, again likely due to the exclusion of crushing loads. 


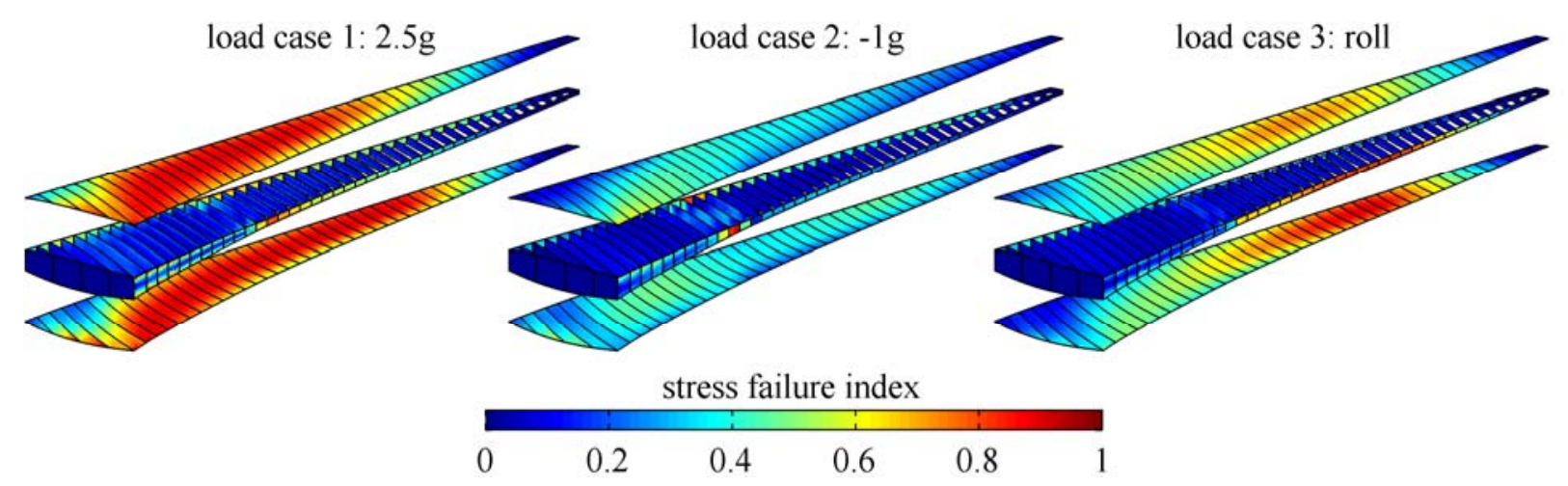

Figure 6. Stress-based failure indices of the optimal design in Figure 4 across each load case.

Panels with negative KS buckling eigenvalue functions are left blank in Figure 7, as this is a physically meaningless result: for a given maneuver case, the loads would have to be entirely reversed for these panels to buckle. This clearly happens through most of the lower surface during the positive load factors (cases 1 and 3 ), and through most of the upper surface during the negative load factor (case 2). Otherwise, most of the panels have a buckling KS factor nearly equal to unity (active constraints) for the upper and lower skins of load case 1 and 2 , respectively, as well as the outboard portion of load case 3 (where the stresses are large). As such, the buckling constraints in Eq. 4 are equally-strong design drivers as compared to the stress constraints, if not stronger. These buckling constraints are also presumably the cause of the large stiffeners utilized in the upper skins (Figure 4), as the 2.5g maneuver causes stronger compressive forces in the upper skin than the -1g maneuver does in the lower skin.

The mass of the optimal structure in Figure 4 is $6,175 \mathrm{~kg}$; this structure is based on the volume of the finite element model (including smeared stiffeners), and does not include the lumped mass or control surfaces seen in Figure 1 and Figure 2. This value can be compared to values obtained below, when additional design variables and aeroelastic constraints are added to Eq. 4.

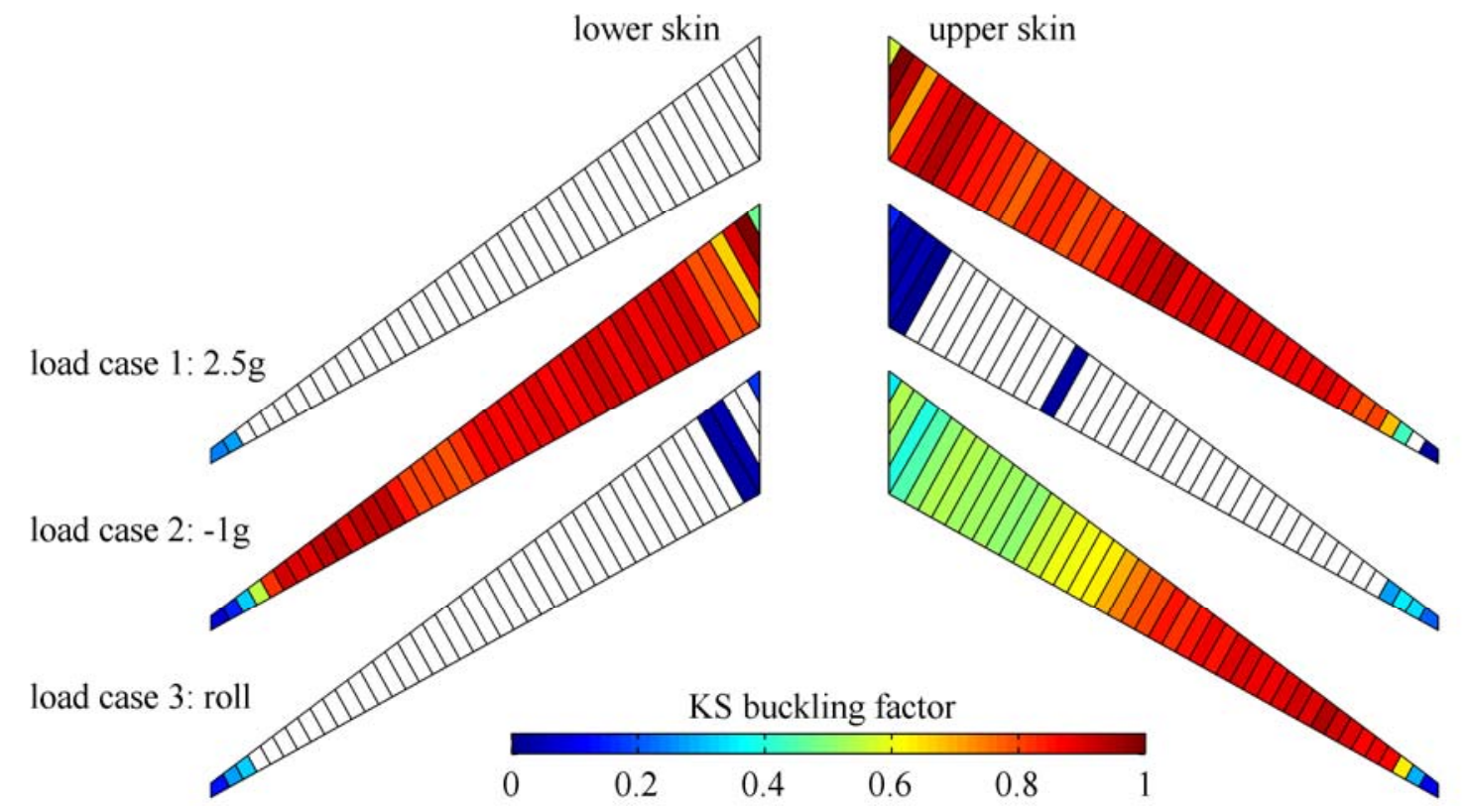

Figure 7. Buckling failure indices (KS functions) for each stiffened panel of the optimal design in Figure 4 across each load case.

\section{Quasi-Steady DMCS Deflection Design Variables}

This section augments the optimization problem of Eq. 4 to include trailing edge flap deflection design variables $\left(\boldsymbol{\gamma}_{s}\right.$ and $\left.\boldsymbol{\gamma}_{a}\right)$, in addition to the structural design variables. Simultaneous handling of wing structure design 
variables and control surface scheduling design variables, within the same optimization loop, is in contrast to most of the existing literature on this topic [5]. Control surface variables are typically computed within an inner loop (over-determined trim optimization), and structural design variables are optimized through an outer loop. In this work, the use of the adjoint method for aeroelastic gradients enables simultaneous optimization of both sets of design variables, which is presumably more efficient. It should be recalled that, whatever control surface deflections are imposed by the optimizer, the aeroelastic analysis (Eqs. 1 and 2) will automatically locate the variables that trim the aircraft ( $\alpha$ and $\delta$ for longitudinal maneuvers, $\alpha$ and $\beta$ for lateral).

As seen in Figure 2, twenty control surfaces are attached to the trailing edge, and for the static aeroelastic analyses considered in this section, the attachments are rigid, and commanded flap rotations are exactly obtained (relative to the deforming wing). The same three load cases are used as in the previous section, but the third load case has both a symmetric $\left(\boldsymbol{\gamma}_{S}\right)$ and an anti-symmetric $\left(\boldsymbol{\gamma}_{a}\right)$ piece, and their scheduling can be optimized separately. This results in 80 total DMCS design variables, in addition to the same structural design variables from above. Side limits of $\pm 10^{\circ}$ are imposed, and the scheduling design variables (as with structural design variables) are passed through a spatial filter in order to maintain a smooth deflection along the trailing edge.

The optimal control surface scheduling is shown in Figure 8, where a positive flap deflection corresponds to a tip-down rotation of the surface. For maneuver load 1 (2.5g pull-up), additional lift is generated inboard, to maintain the overall lift-based trim of the aircraft, but load alleviation is used outboard, in order to reduce the total bending moment on the wing. This allows the optimizer to remove material from the structure (reducing mass, the ultimate objective function) without violating the strength and buckling constraints. Tip-up rotations of all of the control surfaces, from root to tip, would explicitly reduce the stresses and buckling loads even more, but the implicit effect would be an ultimate increase in $\alpha$ to maintain trim, and thus an increase in the aerodynamic loading. It is further noted that many of the $2.5 \mathrm{~g}$ DMCS rotations reside at the side limits of $\pm 10^{\circ}$; further optimization improvement could be expected if these limits were relaxed.

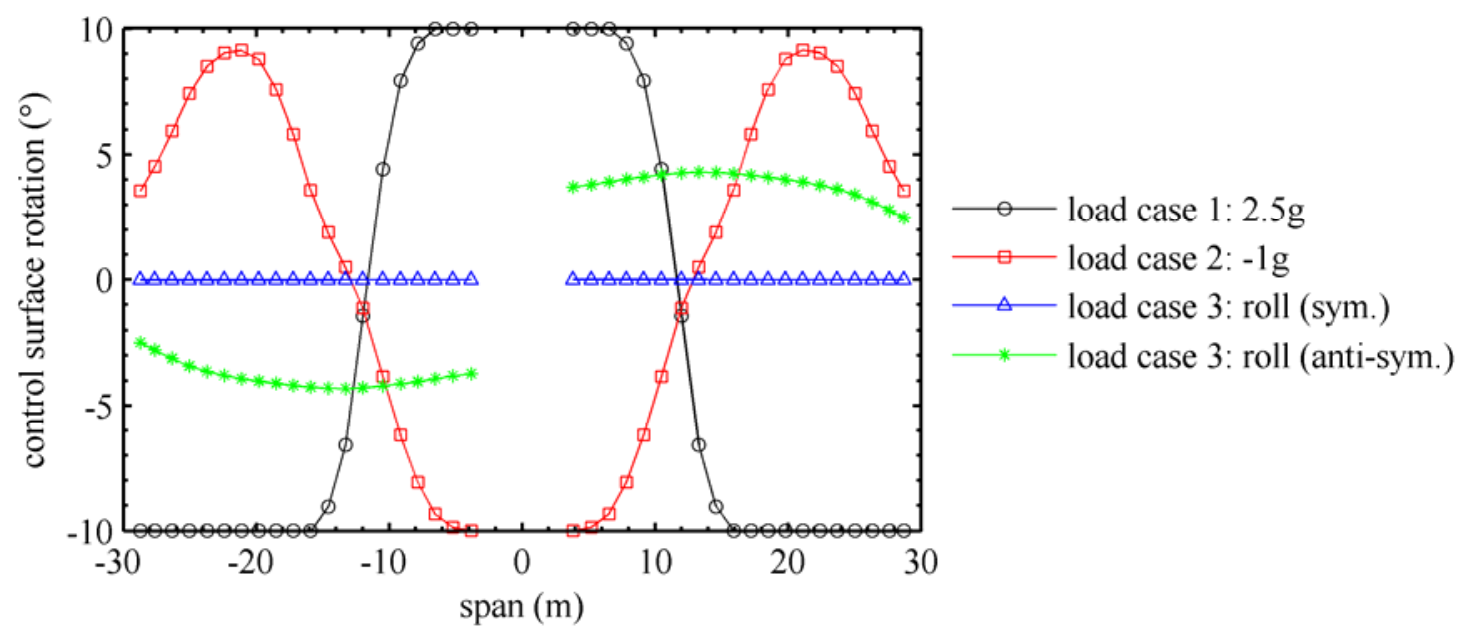

Figure 8. Optimal DMCS rotations for each load case.

Control surface scheduling for load case 2 (-1g push-over) has the expected opposite trends (but lower magnitude) as compared to the $2.5 \mathrm{~g}$ pull-up maneuver. For load case 3 , the symmetric deflections are responsible (along with the angle of attack $\alpha$ ) for maintaining steady level flight $(N=1)$, but these are largely set to zero by the optimizer. The anti-symmetric deflections are responsible for maintaining a steady roll rate $(p \cdot L / U=0.065$, as in all the cases in this paper), and so this is accomplished with a moderate tip-down rotation of all the control surfaces (of the right wing), with peak amplitude near the wing break. Inboard surface rotations are obviously less effective than outboard at generating a rolling moment, but they impose less torsional stress as well, and so the antisymmetric scheduling shown in Figure 8 is the optimal compromise.

The optimal structural design variables (upper and lower skin thickness and stiffener height) for this case are shown in Figure 9, along the span of the wing. This data is directly compared to the optimization case without DMCS design variables, which is repeated from Figure 4. The optimizer is able to significantly decrease the skin thickness, particularly in the upper skins (relative to the case without DMCS variables), though the reduction in stiffener height is milder. The mass of this wing is $4,242 \mathrm{~kg}$, a substantial drop (31.3\%) relative to the structure in Figure 4. 

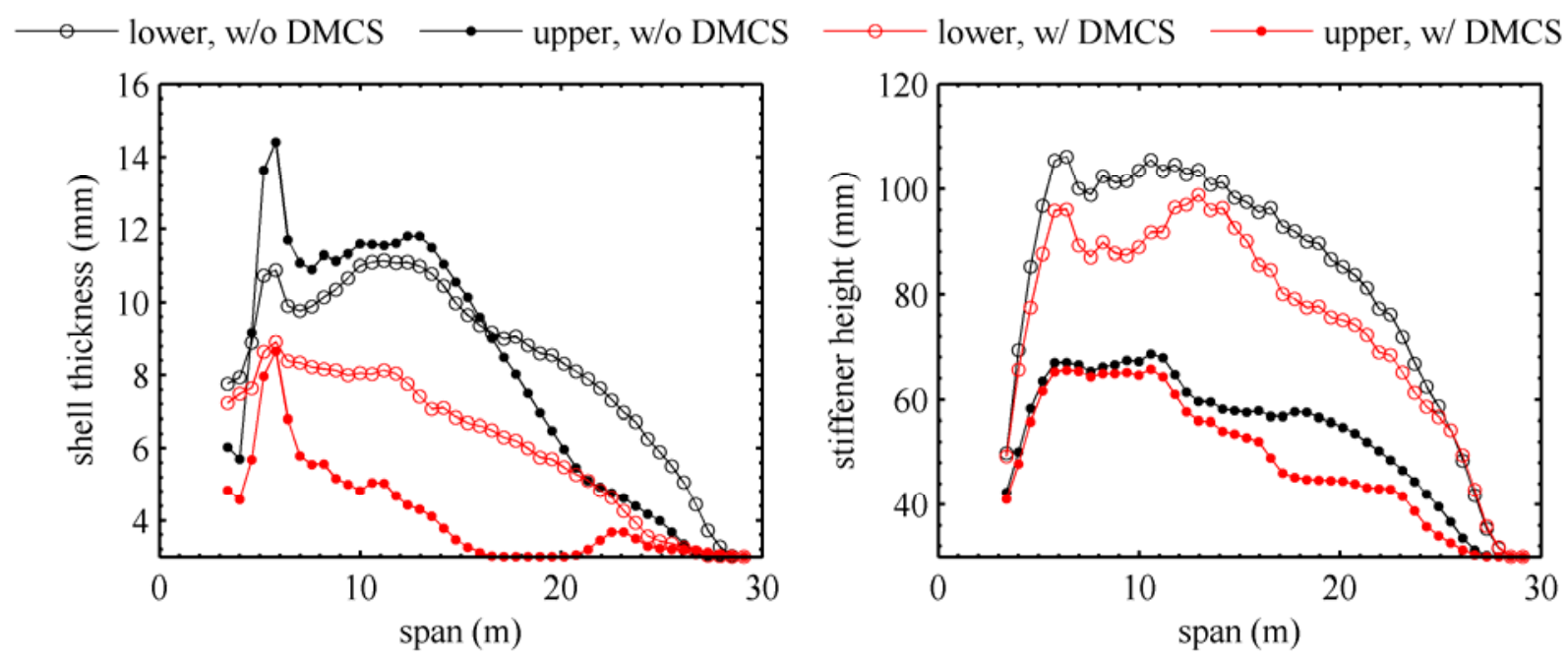

Figure 9. Optimal structural design variables (in the upper and lower skins) with and without DMCS design variables added to the optimization problem.

\section{Open Loop Flutter Constraints}

The exercises in Sections IV and V have only included static aeroelastic considerations (stresses and buckling arising from trim loads), and will now be augmented with an open loop flutter constraint. This constraint stipulates that the wing not flutter within the flight envelope, including an additional margin based upon equivalent air speed. Flutter analysis is conducted using the same configuration seen in Figure 1, Figure 2, and Figure 3, though the aerodynamic paneling of the fuselage, tail, and engine are neglected. Furthermore, each control surface is assumed to be attached to the wing box with a flexible hinge spring.

Aerodynamic analysis is computed using the doublet lattice method in the frequency domain. Open loop flutter constraints could also be computed in the frequency domain (using the p-k method), but are instead computed in the time domain (using the p-method). This is to provide a direct comparison with the results of the next section, where closed loop control requires state space modeling. The development of a state space aeroelastic flutter model is done with well-known methods [2], and will only be briefly described here. First, mode shapes (eigenvectors) of the flexible wing with rigidly-attached control surfaces are computed. Next, twenty control modes (one for each control surface) are computed as Ritz vectors, where each vector is zero except for a unit rotation of the corresponding control surface. The flexible mode shapes and the Ritz vectors are combined into a single modal matrix, and properly orthogonalized [25]. These modes are used to compute reduced mass, stiffness, and damping matrices, as well as generalized aerodynamic forces at tabulated reduced frequencies and Mach numbers. Frequency domain aerodynamics are converted to the time domain using the Roger approximation [26].

The final linear time invariant system is written as:

$$
\left\{\begin{array}{c}
\dot{\boldsymbol{x}} \\
\ddot{\boldsymbol{x}} \\
\dot{\boldsymbol{x}}_{A}
\end{array}\right\}=\left[\begin{array}{ccc}
\boldsymbol{I} & \mathbf{0} & \mathbf{0} \\
\mathbf{0} & \overline{\boldsymbol{M}}-\frac{q \cdot b^{2}}{U^{2}} \cdot \boldsymbol{A}_{2} & \mathbf{0} \\
\mathbf{0} & \mathbf{0} & \frac{b}{U} \cdot \boldsymbol{I}
\end{array}\right]^{-1} \cdot\left[\begin{array}{ccccc}
\mathbf{0} & \boldsymbol{I} & \mathbf{0} & \cdots & \mathbf{0} \\
-\overline{\boldsymbol{K}}+q \cdot \boldsymbol{A}_{0} & -\overline{\boldsymbol{C}}+q \cdot \boldsymbol{A}_{1} & q \cdot \boldsymbol{A}_{2} & \cdots & q \cdot \boldsymbol{A}_{n+2} \\
\mathbf{0} & \frac{b}{U} \cdot \boldsymbol{I} & -\gamma_{1} \cdot \boldsymbol{I} & \cdots & \mathbf{0} \\
\vdots & \vdots & \vdots & \ddots & \vdots \\
\mathbf{0} & \frac{b}{U} \cdot \boldsymbol{I} & \mathbf{0} & \cdots & -\gamma_{n} \cdot \boldsymbol{I}
\end{array}\right] \cdot\left\{\begin{array}{c}
\boldsymbol{x} \\
\dot{\boldsymbol{x}} \\
\boldsymbol{x}_{A}
\end{array}\right\}
$$

where $b$ is half the mean aerodynamic chord, and $\overline{\boldsymbol{M}}, \overline{\boldsymbol{K}}$, and $\overline{\boldsymbol{C}}$ are the reduced mass, stiffness, and damping matrices. $\boldsymbol{A}_{i}$ are the generalized aerodynamic forces resulting from the rational function approximation, and $\gamma_{i}$ are the corresponding lag roots ( $n$ total). $\boldsymbol{x}$ is the vector of modal amplitudes (wing deformation and control surface rotation), and $\boldsymbol{x}_{A}$ is the vector of aerodynamic states. Eigenvalues of Eq. 5 are given by $s=g+i \cdot \omega$. For a given Mach number, these eigenvalues may be tracked across a range of matched point equivalent air speeds. Speeds at which a given eigenvalue crosses into the right-half plane $(g=0)$ are flutter points. Any flutter point that occurs within the vicinity of the flight envelope by less than a $20 \%$ margin (as measured by equivalent air speed) is considered unacceptable. 
The method of Ringertz [27] is used to formulate a flutter constraint, where the damping, $g$, of each mode is constrained to lie below a given curve at all equivalent air speeds $U_{E A S}$ of interest:

$$
g \leq\left\{\begin{array}{lc}
0 & 0 \leq U_{E A S}<U^{*} \\
s \cdot\left(U_{E A S}-U^{*}\right)^{2} & U_{E A S} \geq U^{*}
\end{array}\right.
$$

where $s$ is a scaling parameter, and $U^{*}$ is the minimum allowable flutter equivalent air speed. Critical $U_{E A S}$ points (local minima) of the inequality in Eq. 6 are computed and lumped together into a single Kreisselmeier-Steinhauser constraint. As above, if this constraint is greater than one, then Eq. 6 is not satisfied, and the structure does not meet the required flutter margin. A separate KS constraint is utilized for each considered Mach number. Derivatives of the flutter constraint are easily computed with eigenvalue gradient techniques. A free-mode dynamic derivative approach is utilized here, where the derivative of the mode shapes with respect to design variables is neglected for the purposes of gradient computations.

The optimization problem of the previous section is repeated here, adding the flutter constraint (at 0.85 Mach) to the static aeroelastic buckling and stress constraints. Both structural design variables and quasi-steady DMCS design variables are utilized as before. Several optimal results are presented in Figure 10 for various required flutter margins, and for two different DMCS hinge spring frequencies: 9 and $25 \mathrm{~Hz}$. For reference, the first bending frequency of the optimal wing structures is on the order of $1 \mathrm{~Hz}$. The dashed line in the figure indicates the minimum mass, which may be obtained with no flutter constraint: $4,242 \mathrm{~kg}$, obtained in the previous section. As expected, enforcing a flutter constraint increases the optimal mass. For the $25 \mathrm{~Hz}$ hinge spring case (for which the control surfaces are nearly rigidly attached), the mass penalty is $34.4 \%$ under a $20 \%$ margin, which is typically required for civilian aircraft. For the more flexible hinge case, the penalty is nearly a factor of three.

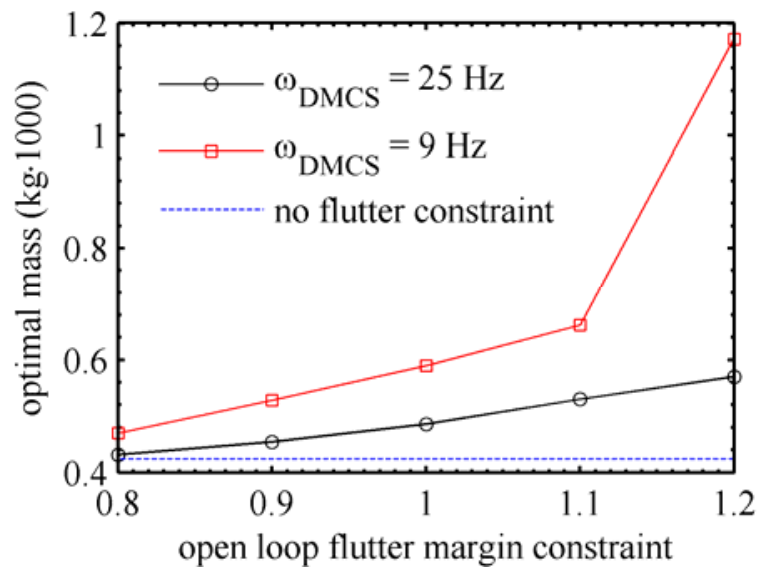

Figure 10. Pareto front between the flutter constraint boundary and the minimum structural mass.

The aggressive flutter mechanism (and resulting mass penalty) associated with a flexible hinge spring is well known [28], and results from a situation where coupling between wing motion and control surface rotation exacerbates aerodynamic forces. More details are given in Figure 11, which tracks the various flutter mechanisms across a range of DMCS frequencies. This is done for the wing structure optimized without a flutter constraint. Three instabilities are seen for higher hinge frequencies, and the flutter speeds approach the values that would be obtained with a rigid hinge connection. These three flutter mechanisms become more severe (lower $U_{E A S}$ ) as the hinge spring frequency is decreased, and several additional mechanisms appear as well. The flutter speeds of these new mechanisms drop rapidly with decreased hinge frequency. At the lowest hinge frequency considered $(7 \mathrm{~Hz})$, the critical flutter speed is almost a third the value of the critical flutter speed computed with a rigid control surface assumption.

Eigenvalue migration plots are shown in Figure 12 before and after the 20\% flutter margin constraint is implemented, for a hinge spring frequency of $25 \mathrm{~Hz}$. Similar data is given in Figure 13 for the lower value of $9 \mathrm{~Hz}$. The top row of plots in both figures corresponds to the dashed line in Figure 10 (no flutter constraint), while the bottom row of plots corresponds to the data points with the largest mass in Figure 10. At the higher hinge frequency (Figure 12), the three flutter mechanisms noted in Figure 11 are seen here as well: two are hump modes, the other is a more severe instability. This is commonly seen for transport aircraft [29], where the primary hump mode is a 
torsion mode dominated by engine pitch (mode 2), and the "hard" flutter point is a bend-torsion coupling. The dashed line in these figures is the constraint boundary of Eq. 6. This constraint is satisfied in the lower row of plots in Figure 12, and strongly active, as two modes brush up against the boundary in the vicinity of $U^{*}$.
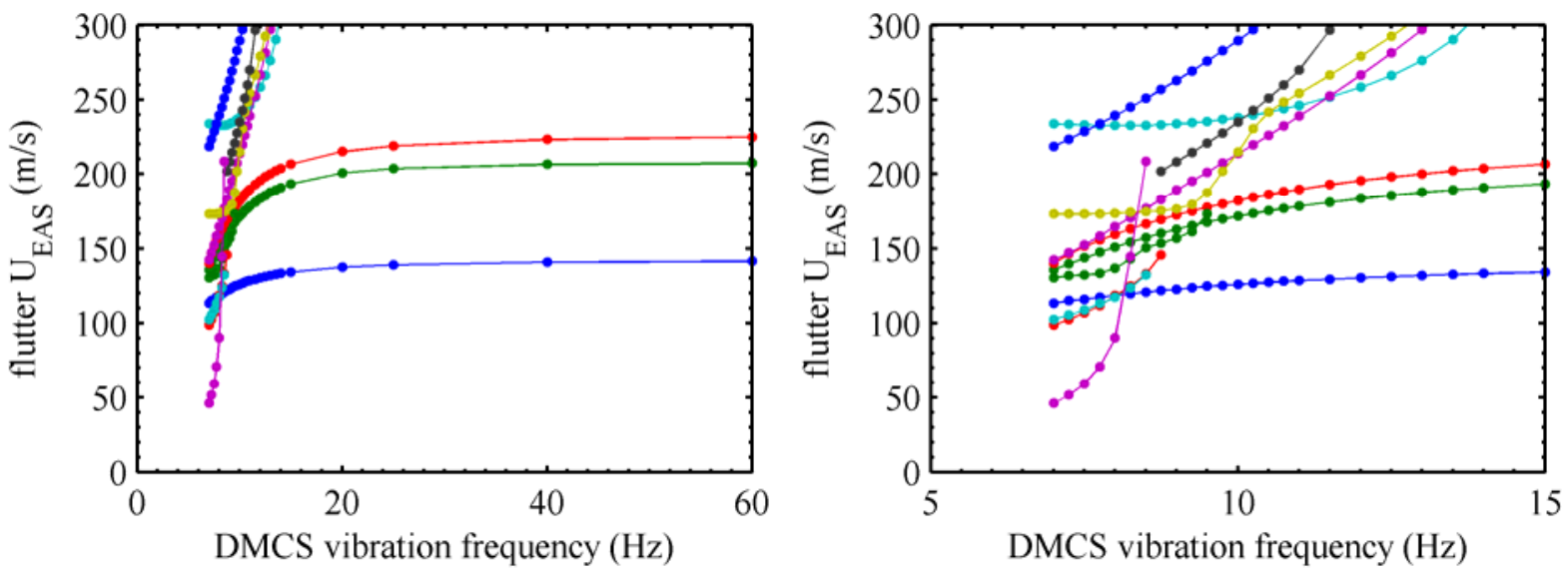

Figure 11. Influence of control surface hinge frequency on the flutter mechanism.
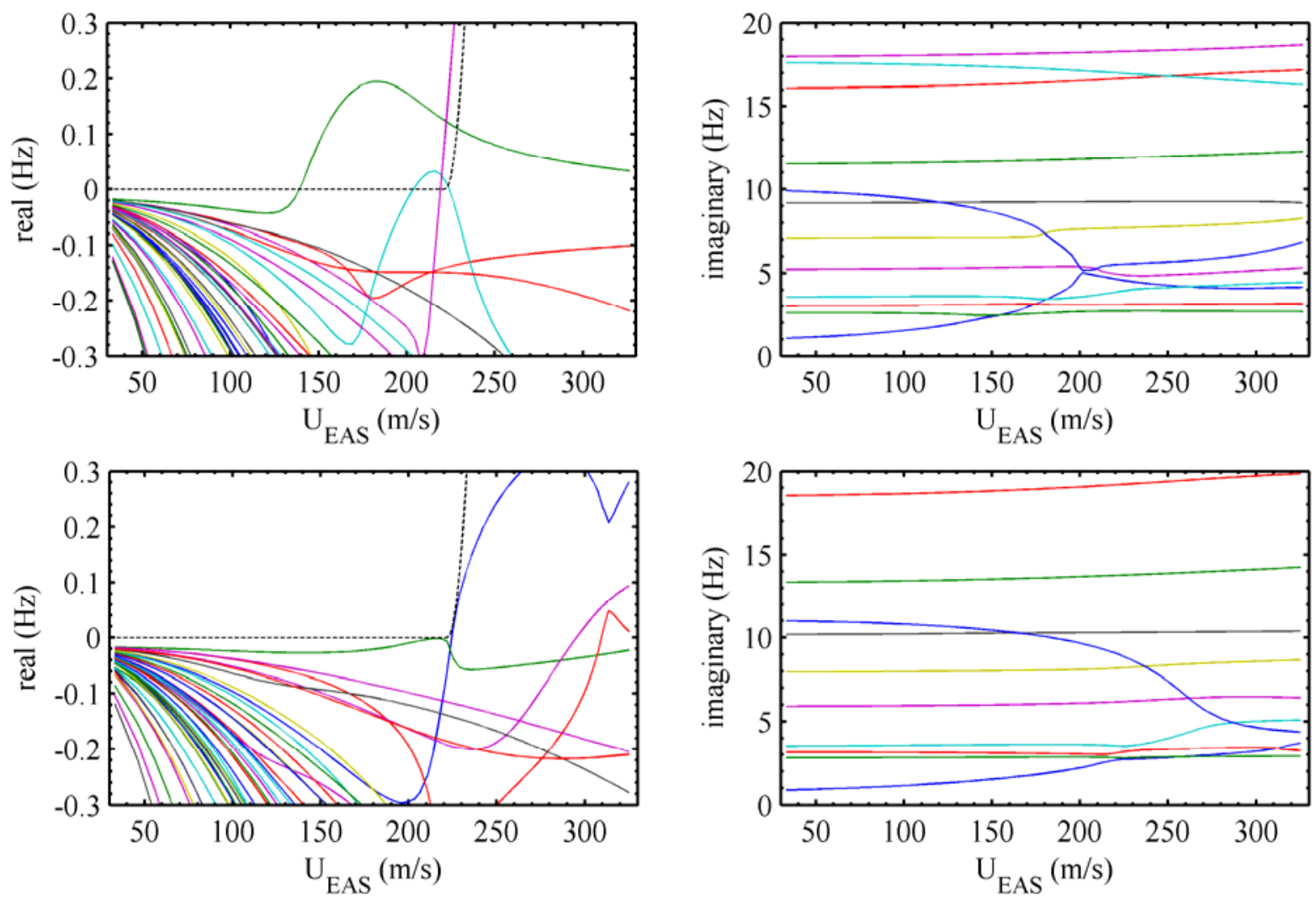

Figure 12. Eigenvalue migration without (top row) and with (bottom row) a $20 \%$ flutter margin constraint enforced: $25 \mathrm{~Hz}$ DMCS frequency.

For the lower stiffness hinge spring (9 Hz, Figure 13), multiple hump mode and hard crossing flutter mechanisms are seen. The optimizer is also able to push each of these fluttering modes beyond the constraint boundary (lower row of plots), by adding a substantial amount of structure to the wing, as seen in Figure 10. Four separate modes are active in this case, but do not brush right up against the constraint boundary as seen in Figure 12. This is due to the conservatism of the aggregate KS constraint, which becomes more pronounced as more failure 
mechanisms engage. Finally, the twenty $9 \mathrm{~Hz}$ modes (one for each DMCS) are also noted in the imaginary eigenvalue migration plots of Figure 13.
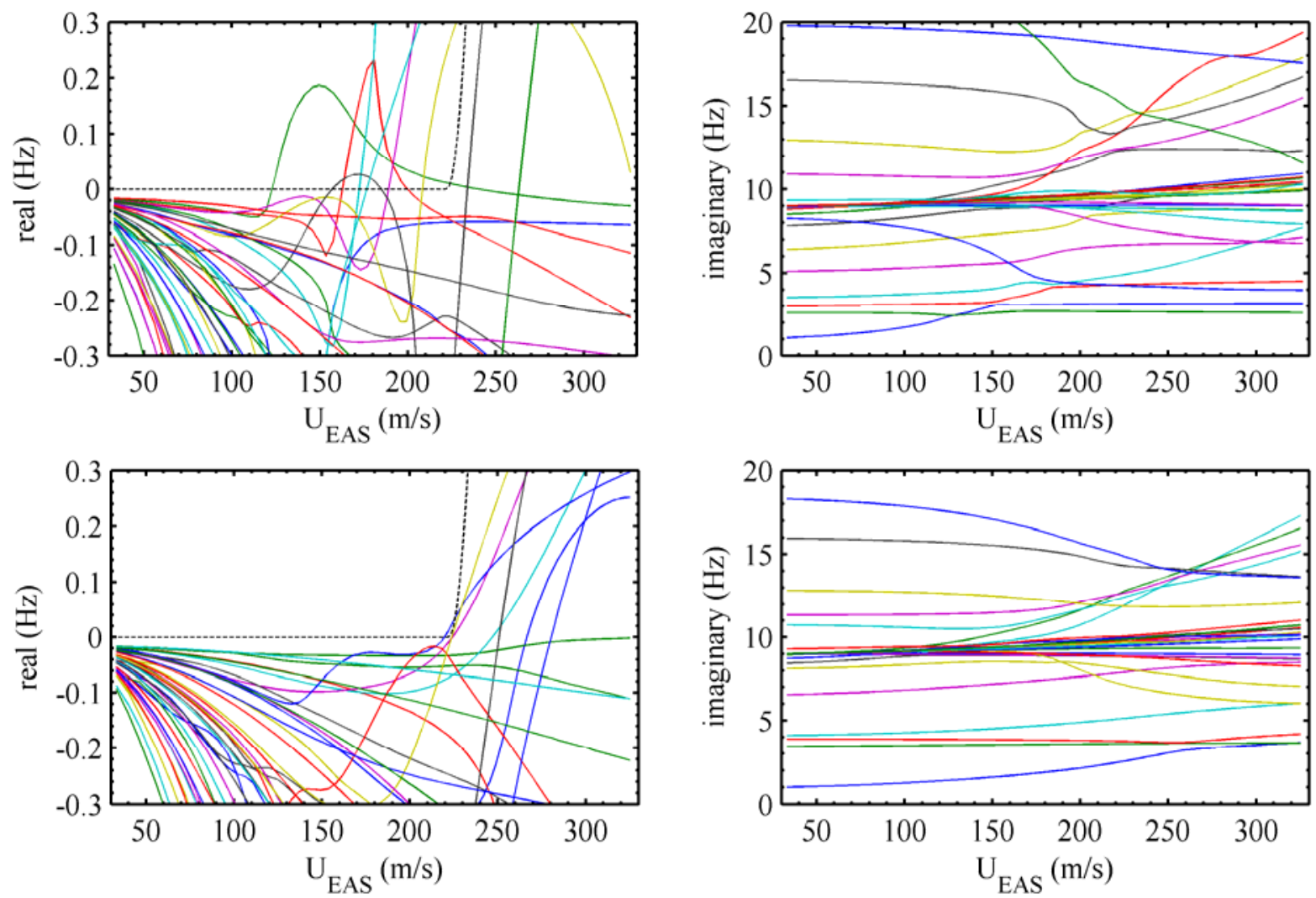

Figure 13. Eigenvalue migration without (top row) and with (bottom row) a $20 \%$ flutter margin constraint enforced: 9 Hz DMCS frequency.

Optimal structural design variables for the case with $25 \mathrm{~Hz}$ hinge springs and 20\% imposed flutter margin are seen in Figure 14. Several key topological differences are noted here, as compared to the static aeroelastic optimum in Figure 4. Most predominantly, material is added to the wing tip leading edge, a clear attempt to alter the inertial loads via separation of coalescing modal frequencies. It is noted that landing loads and taxi bump loads are not included in the list of load cases here, but these types of inertial forces would penalize the tip mass [30] seen in Figure 14, and force the optimizer to be less reliant on this form of flutter mitigation. Secondly, the thickness of the spars is increased in the vicinity of the engine attachment points, which is presumably driven by the critical engine pitch fluttering hump mode. Finally, the optimizer is able to push most of the stiffener height design variables along the lower skin to the lower bound. The reason for this is unclear (large stiffeners are needed for the static aeroelastic case in Figure 4), though perhaps the added stiffness required to alleviate the flutter mechanism has implicitly decreased the need for large stiffeners as well.

\section{Closed Loop Flutter Constraints}

It is seen in Section VI that an open loop flutter constraint can be satisfied using structural and quasi-steady DMCS design variables, though a weight penalty is imposed as well. For largely-rigid hinge spring connections (25 $\mathrm{Hz}$ ), the penalty is $34.4 \%$ under a $20 \%$ margin, and this penalty increases for more-flexible springs. This section explores the idea of relaxing the open loop flutter constraint, relying on closed loop control to meet the $20 \%$ margin, and assessing the weight savings from such a shift. At its most extreme, the open loop flutter constraint may be entirely removed, though this leads to a potentially dangerous situation where the vehicle relies on a controller to prevent a catastrophic aeroelastic instability within its flight envelope. More realistically (from a certification standpoint), the open loop margin may be relaxed to only $5 \%$ or $10 \%$. Both cases will be considered here. Ideally, 
the controller may be able to meet this flutter margin without needing to add extra stiffness (mass) to the wing, though the control cost (system power) may be excessive [2].

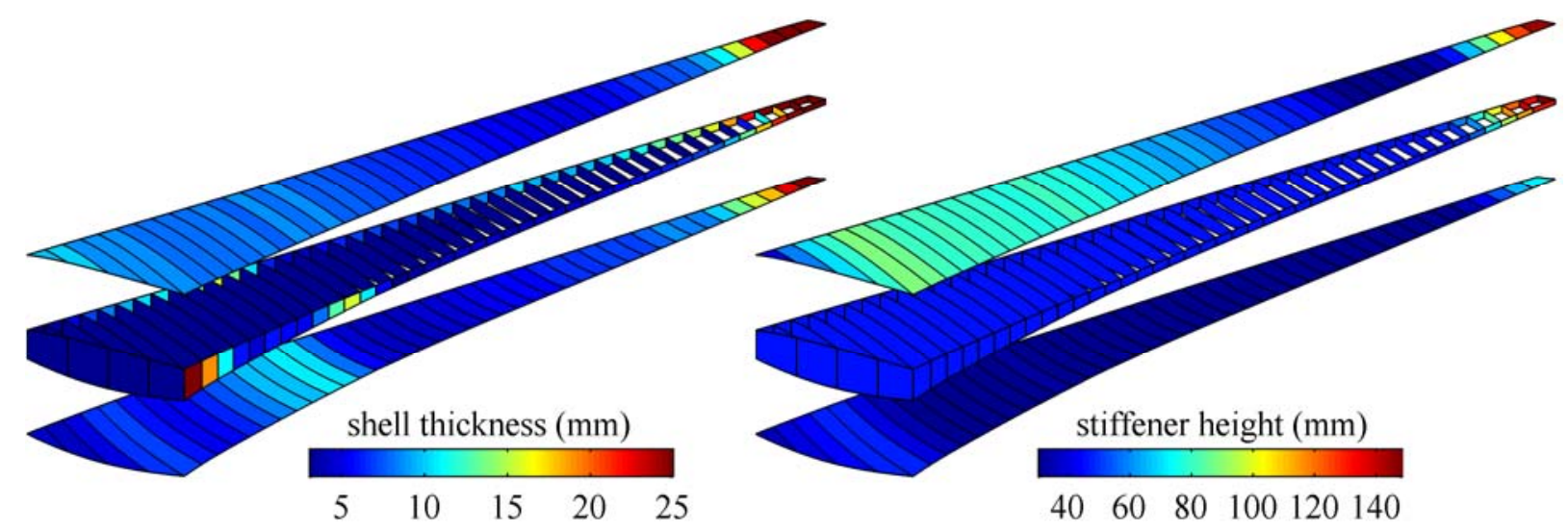

Figure 14. Optimal structural design variables for the optimization problem with static aeroelastic constraints and open loop flutter constraints.

The aeroservoelastic linear time invariant system is given as:

$$
\dot{\boldsymbol{x}}_{A S E}=\boldsymbol{A} \cdot \boldsymbol{x}_{A S E}+\boldsymbol{B} \cdot \boldsymbol{u}_{A S E}
$$

where $\boldsymbol{x}_{A S E}$ is the state vector from Eq. 5, $\boldsymbol{A}$ is the matrix from Eq. 5, $\boldsymbol{u}_{A S E}$ is a vector of input hinge moments (one per control surface), and $\boldsymbol{B}$ is a matrix which converts those hinge moments into generalized modal forces. The controller used here is a simple linear quadratic regulator (LQR), where feedback is assumed of the form:

$$
\boldsymbol{u}_{A S E}=-\boldsymbol{K} \cdot \boldsymbol{x}_{A S E}
$$

The feedback matrix is found which minimizes the performance index [31]:

$$
\boldsymbol{J}=\frac{1}{2} \cdot \int_{0}^{\infty}\left(\boldsymbol{x}_{A S E}^{T} \cdot \boldsymbol{Q} \cdot \boldsymbol{x}_{A S E}+\boldsymbol{u}_{A S E}^{T} \cdot \boldsymbol{R} \cdot \boldsymbol{u}_{A S E}\right) \cdot d t
$$

where $\boldsymbol{Q}$ is the state weighting matrix, and $\boldsymbol{R}$ is the control weighting matrix.

Two types of controller-centric design variables are considered here. The first are the members of the diagonal control weighting matrix $\boldsymbol{R}$ (one per control surface), which are passed through a spatial filter in order to maintain a smooth oscillatory shape along the trailing edge, much like the quasi-steady DMCS design variables. Upper and lower bounds are necessarily placed on the $\boldsymbol{R}$ design variables. Very large values (relative to $\boldsymbol{Q}$, which is a fixed quantity for all cases in this paper) penalize the control action, and so instabilities will be arrested slowly. Very small values of $\boldsymbol{R}$ are indicative of cheap control, where instabilities can be damped out quickly.

The second type of controller design variable is the flight condition (namely, the equivalent air speed $U_{L Q R}$ ) at which the feedback matrix $\boldsymbol{K}$ is computed. Gain scheduling is the preferred long-term solution for active flutter control of transport wings [32], but for the conceptual studies provided here, a single control law is used for the entire flight envelope. The optimizer is able to choose the flight condition at which the controller is designed, however. A similar type of design variable is utilized in Refs. [6], [9], and [10]. Large values of the $U_{L Q R}$ control design point (relative to the open loop flutter point) will stabilize the system at $U_{L Q R}$, as guaranteed by the LQR methodology [31], but the control cost $\boldsymbol{J}$ will be high. Small values of $U_{L Q R}$ may not provide a closed loop flutter point measurably better than the open loop point, but the control cost will be low.

The aeroservoelastic optimization problem solved in this section is to minimize mass, subject to the static aeroelastic stress and buckling constraints, an open loop flutter constraint, a closed loop flutter constraint, and a limit on the maximum allowable control cost $\boldsymbol{J}$. The closed loop constraint is always set to a margin of $20 \%$, but lower open loop margins are considered, as discussed above. Design variables include structural variables (shell thickness, stiffener thickness, stiffener height), quasi-steady DMCS rotation variables, the control weighting terms $\boldsymbol{R}$, and the 
controller design point $U_{L Q R}$. Gradients of the feedback matrix $\boldsymbol{K}$ and the performance index $\boldsymbol{J}$ are computed using methods in Ref. [33]. Once the $\boldsymbol{K}$ derivatives are found, derivatives of the closed loop flutter constraint are computed with the same eigenvalue sensitivity methods used for the open loop constraint.

As with the open loop flutter constraint, a single Mach number of 0.85 is considered for the closed loop constraint. Furthermore, only the stiffer control surface hinge spring frequencies of $25 \mathrm{~Hz}$ are considered for this section. Prior to presented optimization results, trade studies demonstrating the dependence of the aeroservoelastic behavior upon $\boldsymbol{R}$ and $U_{L Q R}$ are conducted. This is done for the static aeroelastic optimum found in Section $\mathrm{V}$, with a mass of 4,242 kg. Figure 15 shows the closed loop flutter speed and the control cost for different values of $\boldsymbol{R}$ (the same fixed value for each control surface input). As indicated in the figure, the controller is designed at an unstable $U_{L Q R}$ value $11.9 \%$ beyond the open loop flutter point. The LQR controller is guaranteed to stabilize the system at the design speed $U_{L Q R}$, even for very expensive controllers. For the highest values of $\boldsymbol{R}$, the closed loop flutter speed is $35.2 \%$ higher than the open loop value.
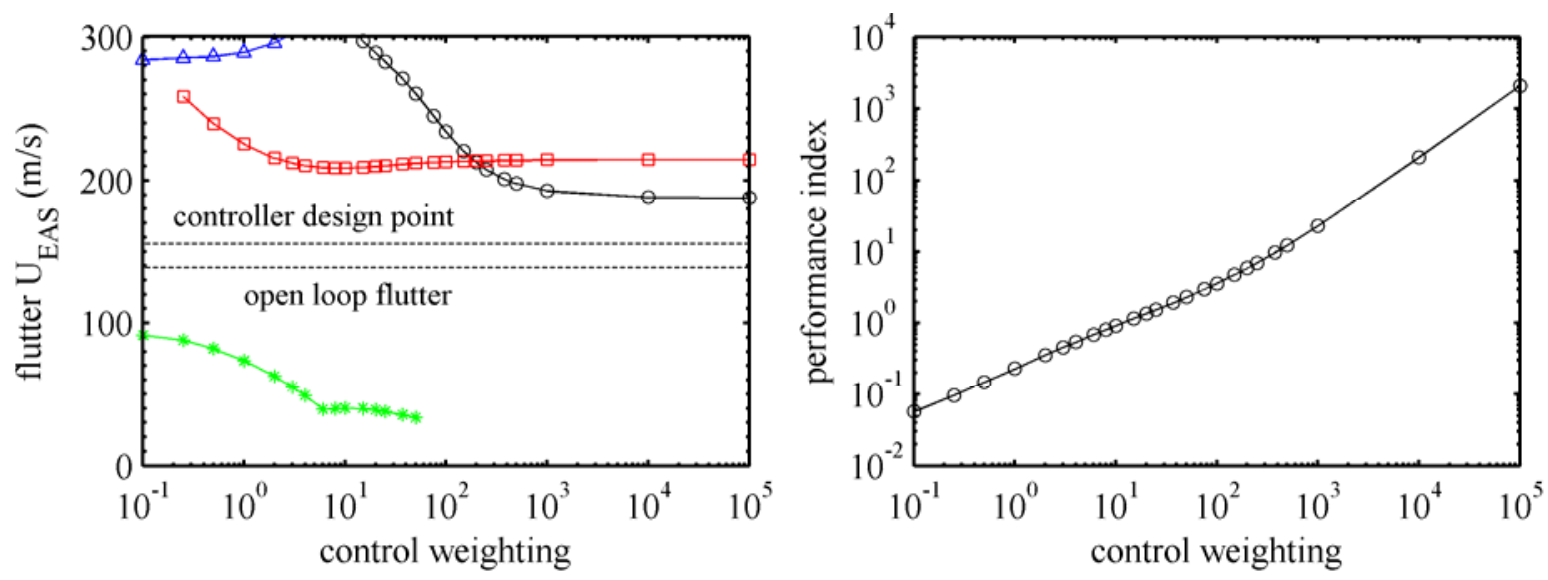

Figure 15. Dependence of closed loop flutter speed and LQR performance index upon weighting matrix $R$.

For lower values of $\boldsymbol{R}$ in Figure 15, a higher premium is placed upon quickly arresting instabilities: substantially higher closed loop flutter points are seen in this area, nearly twice the open loop value. A caveat to this result (beyond the questionable idea of highly inexpensive control) is that some of the wind-off modes are unstable, as seen in the lower locus of flutter points in Figure 15. These modes re-stabilize (at a point not shown in the figure) as the equivalent air speed is increased to $U_{L Q R}$, as guaranteed by the LQR methodology. Similar behavior is noted in Ref. [9] and [34], and is presumably due to the stabilizing aerodynamic forces utilized by the controller at $U_{L Q R}$, but which are not present at very low dynamic pressures. A long-term solution to this problem is gain scheduling, which is not utilized here. Alternatively, the upper and lower bounds on $\boldsymbol{R}$ are (arbitrarily but necessarily) set at 50 and 700, where the value of 50 is high enough that unstable wind-off modes do not appear during the optimization process.

Similar data is provided in Figure 16 for changes in the LQR design condition $U_{L Q R}$, for fixed $\boldsymbol{R}$ values of 200. For the left plot, the system is always closed loop stable along the line with unit slope. For values of $U_{L Q R}$ well beyond the open loop flutter point, the control cost $\boldsymbol{J}$ is very large and instabilities appear at lower equivalent air speeds, through a mechanism very similar to the previous figure. At very low values of $U_{L Q R}$, the closed loop flutter point approaches the open loop value, as the aeroelastic interactions that are used to derive the controller $\boldsymbol{K}$ are too weak to provide additional flutter mitigation beyond what is already available.

Aeroservoelastic optimization results are now presented. Figure 17 shows the Pareto front between the optimal wing mass and the open loop flutter margin. The first curve shows a series of optimization results where only an open loop constraint is included (no control); this data is repeated from Figure 10. Next, the closed loop flutter constraint is set to $20 \%$, and the open loop flutter constraint is progressively relaxed. This curve is coincident with the first, as the controller is able to meet the $20 \%$ closed loop constraint without adding any additional mass to the structure, and also without exceeding the performance index constraint limit, which is set to 10. For the final curve in Figure 17, the performance index limit is much harder to satisfy (set to one), and the LQR controller is unable to stabilize the system at a $20 \%$ margin without exceeding the allowable cost. The optimizer is then forced to add additional structure to the wing, with an $11.3 \%$ mass increase for the most critical case considered of 0.8 (-20\% open 
loop margin). Though not shown, the optimizer also re-adjusts the quasi-steady DMCS control surface rotations. These design variables have no explicit effect on either the wing mass objective or the flutter constraints, but can help maintain static aeroelastic constraints when material is re-allocated to satisfy dynamic constraints.
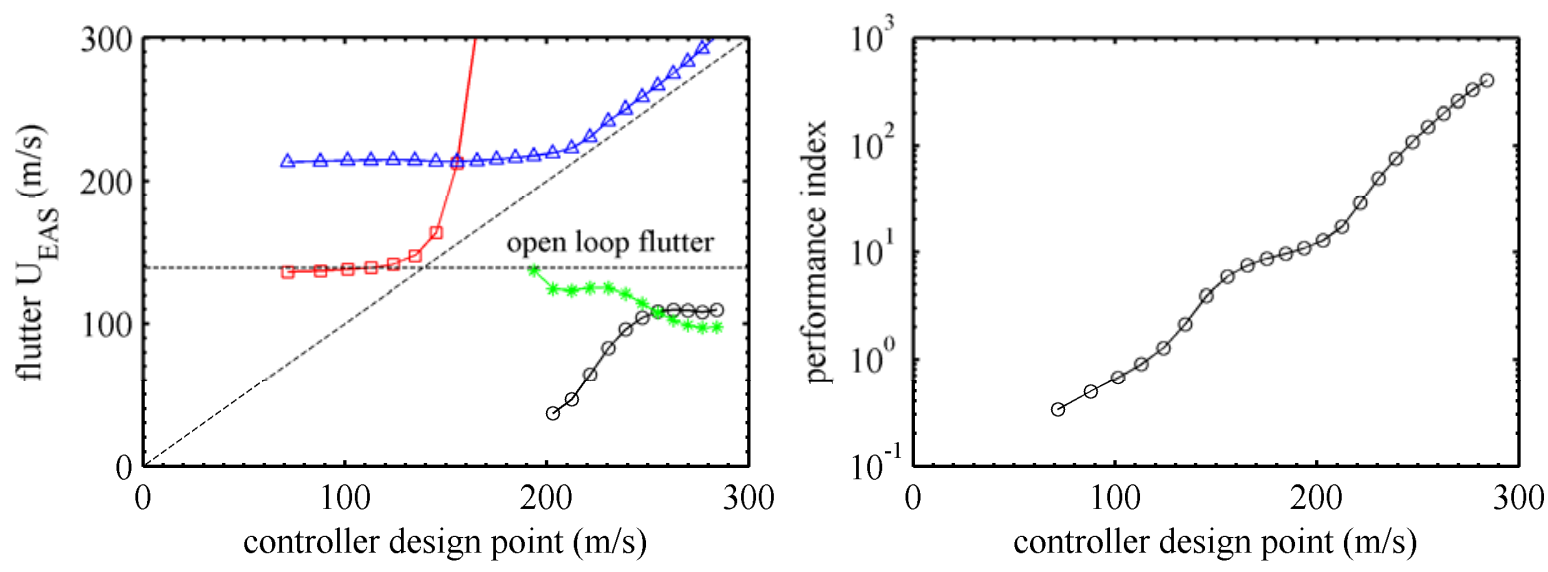

Figure 16. Dependence of closed loop flutter speed and LQR performance index upon the LQR design point.

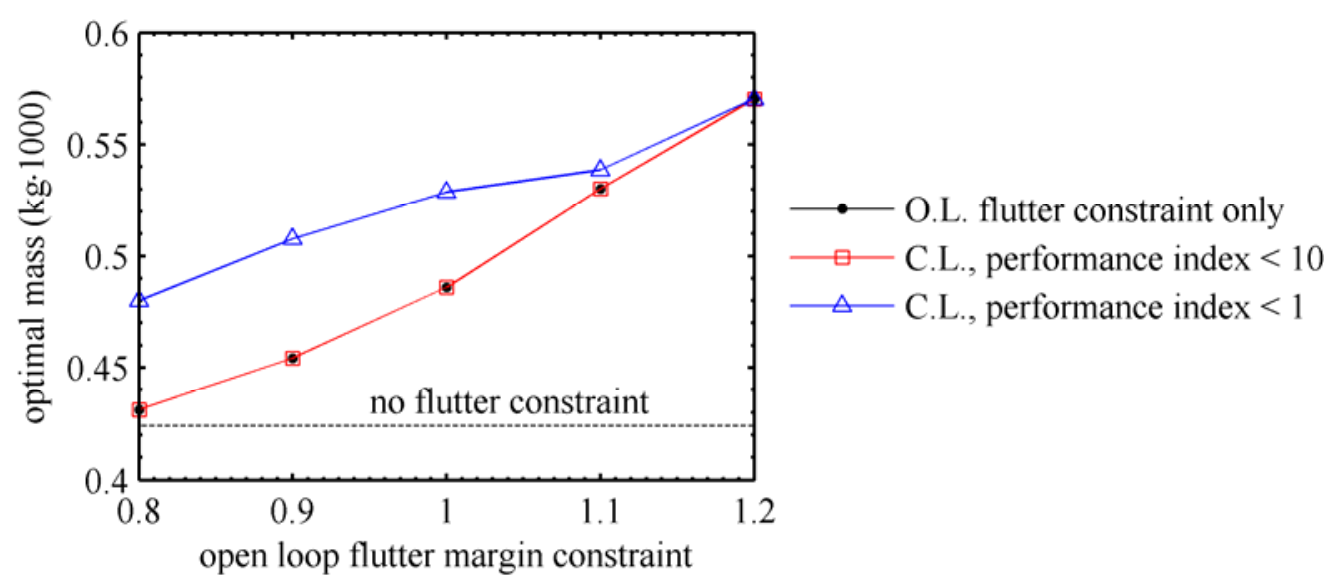

Figure 17. Pareto front between the open loop flutter constraint boundary and the minimum structural mass.

Eigenvalue migration (real parts) for the -20\% open loop margin case in Figure 17 is shown in Figure 18. Both open loop and closed loop eigenvalues are shown, and in both cases the corresponding flutter constraint $(-20 \%$ margin for open loop, $+20 \%$ for closed loop) boundary of Eq. 6 is strongly active. The closed loop case, in particular, has two modes closely interacting with the constraint boundary. Open and closed loop flutter modes (eigenvectors of Eq. 7) for this case are shown in Figure 19. The open loop flutter shape is a combination of wing bending, torsion, and engine pitch, and the control surfaces rigidly follow the trailing edge wing shape. The closed loop flutter shape shows less bending, and the input hinge moments (from the feedback controller) show noticeable control surface rotations relative to the wing, particularly in the region between the engine and the wing tip.

The results of Figure 17 indicate a trade-off between structural mass and control cost, an idea discussed in Ref. [2] also. The complete trade-off curve between these two metrics is shown in Figure 20, where the open and closed loop flutter constraints are set to 0.8 and $1.2(-20 \%$ and +20 margins), respectively. The corresponding control weighting design variables $\boldsymbol{R}$ along the trailing edge are shown in Figure 21. For higher allowable control cost values, the optimizer is able to meet the closed loop flutter constraint without adding additional mass to the wing, as noted in Figure 17 also. The $\boldsymbol{R}$ values for these cases (300) are unchanged from the initial value given to the optimizer, as these design variables are unneeded. Only when the allowable control cost constraint $\boldsymbol{J}$ is decreased below 2 is the optimizer finally forced to add mass to the wing, as this constraint is too restrictive for the LQR controller to satisfy on its own. 

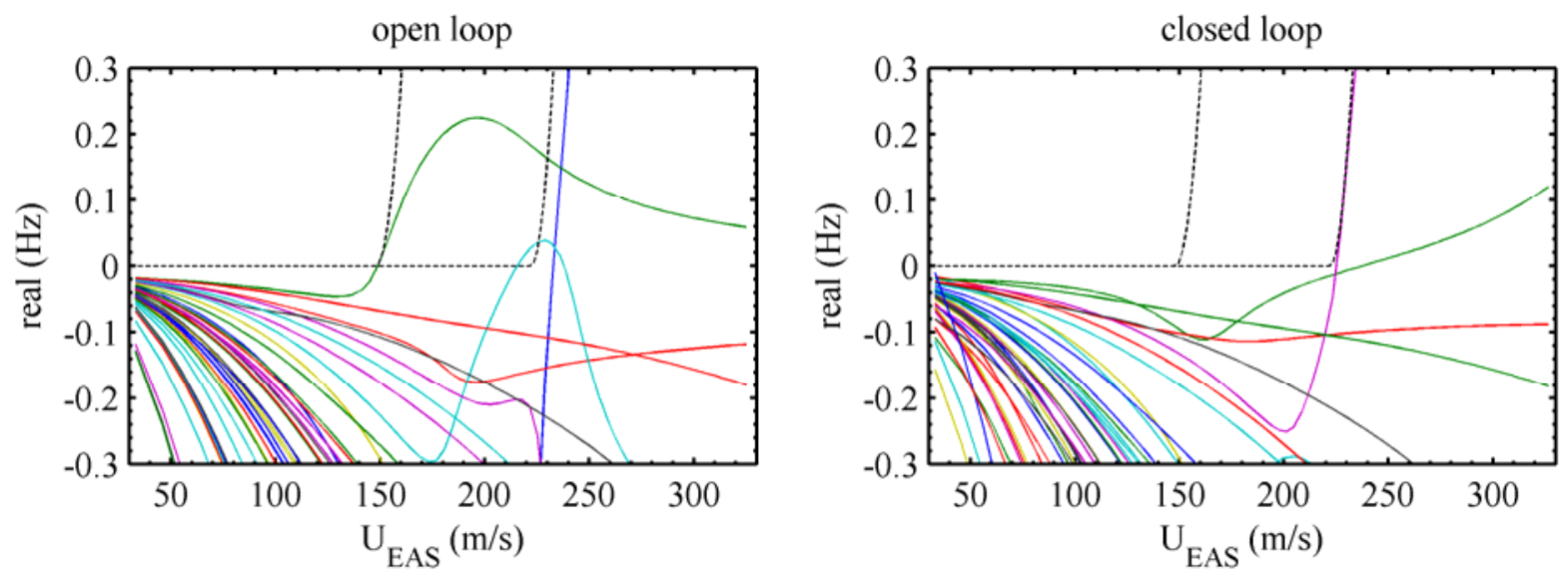

Figure 18. Open (left) and closed loop (right) eigenvalue damping under active flutter constraints.

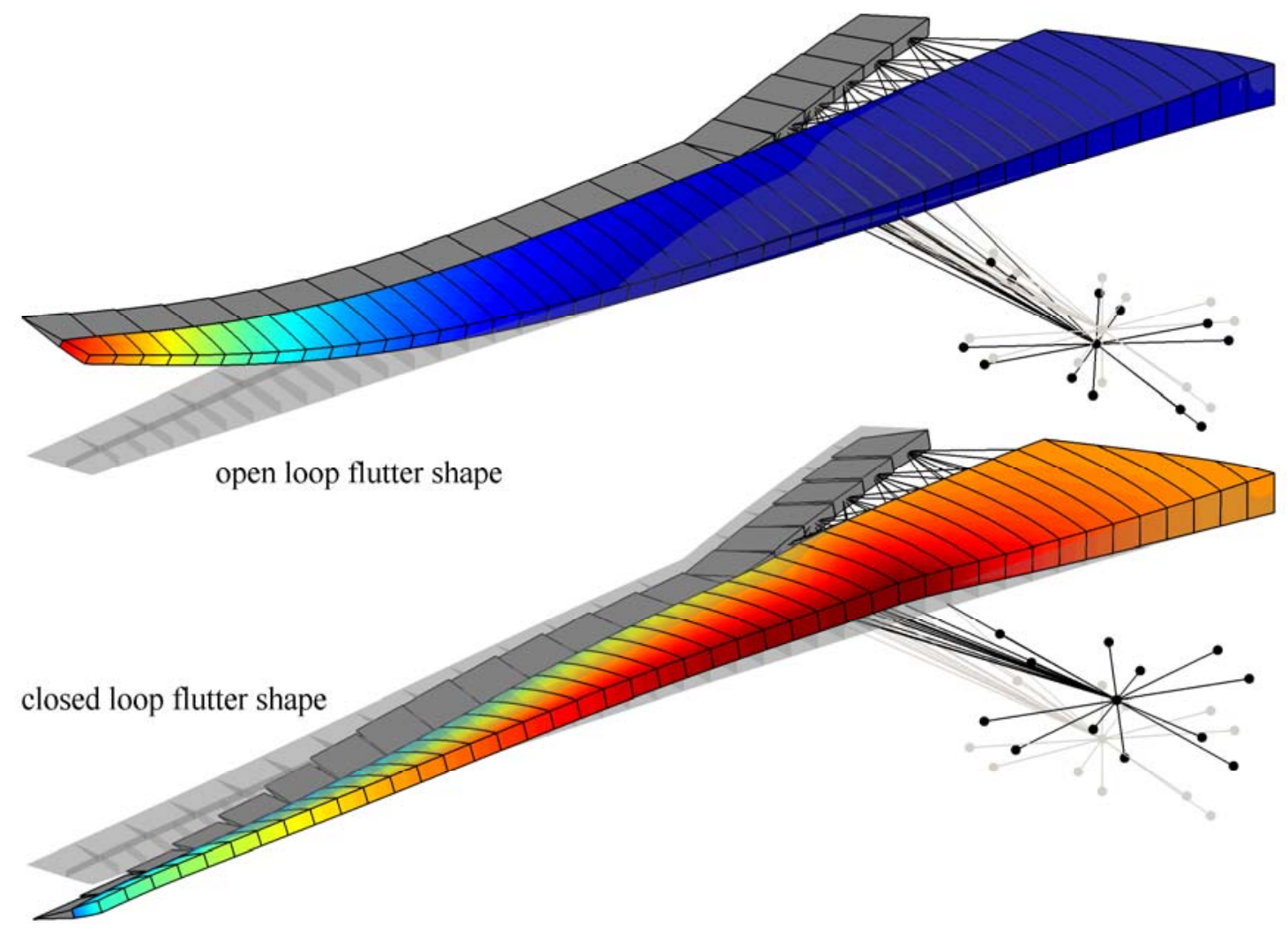

Figure 19. Open (top) and closed (bottom) loop flutter mode shapes.

The expected monotonic increase in optimal wing mass with decreasing control cost requirements is seen in Figure 20 between $\boldsymbol{J}$ thresholds of 2 and 0.5 . $\boldsymbol{R}$ values along the trailing edge are at the lower bound of 50, except near span stations that align with the engine attachment, where the upper bound of 700 is met. This is presumably related to the strong engine pitch noted in the closed loop flutter mode shape of Figure 19. Decreasing the cost constraint below 0.5 leads to strong discontinuities in the aeroservoelastic design space, whose source is ultimately unclear. There are no optimal designs between $\boldsymbol{J}$ thresholds of 0.5 and 0.1438 , and again between 0.1438 and 0.084 . The design at $\boldsymbol{J}=0.1438$ converges very slowly, and the optimization algorithm encounters discontinuities in the closed loop response and controller cost with small changes in the design inputs. This area of the design space is accompanied by a substantial change in the optimal $\boldsymbol{R}$ distribution along the trailing edge (Figure 21), where the inboard control surfaces are all at the upper bound of 700. A monotonic relationship between wing mass and $\boldsymbol{J}$ is 
again seen below 0.1438 , where every control weighting value $\boldsymbol{R}$ is pushed to the lower bound of 50 . This is the expected value for a situation with very-stringent control cost limitations, given the near-proportional relationship between $\boldsymbol{R}$ and $\boldsymbol{J}$ seen in Figure 15.

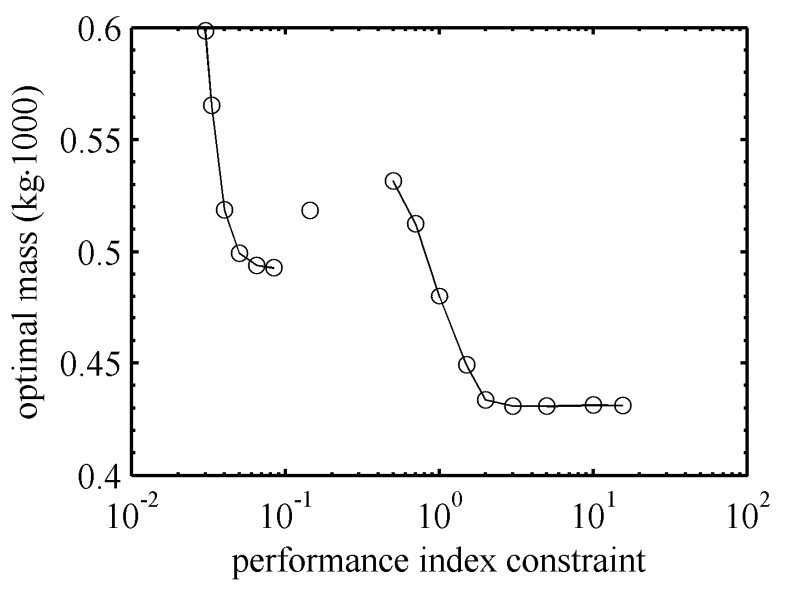

Figure 20. Trade-off between the allowable LQR control cost and the minimum structural mass.

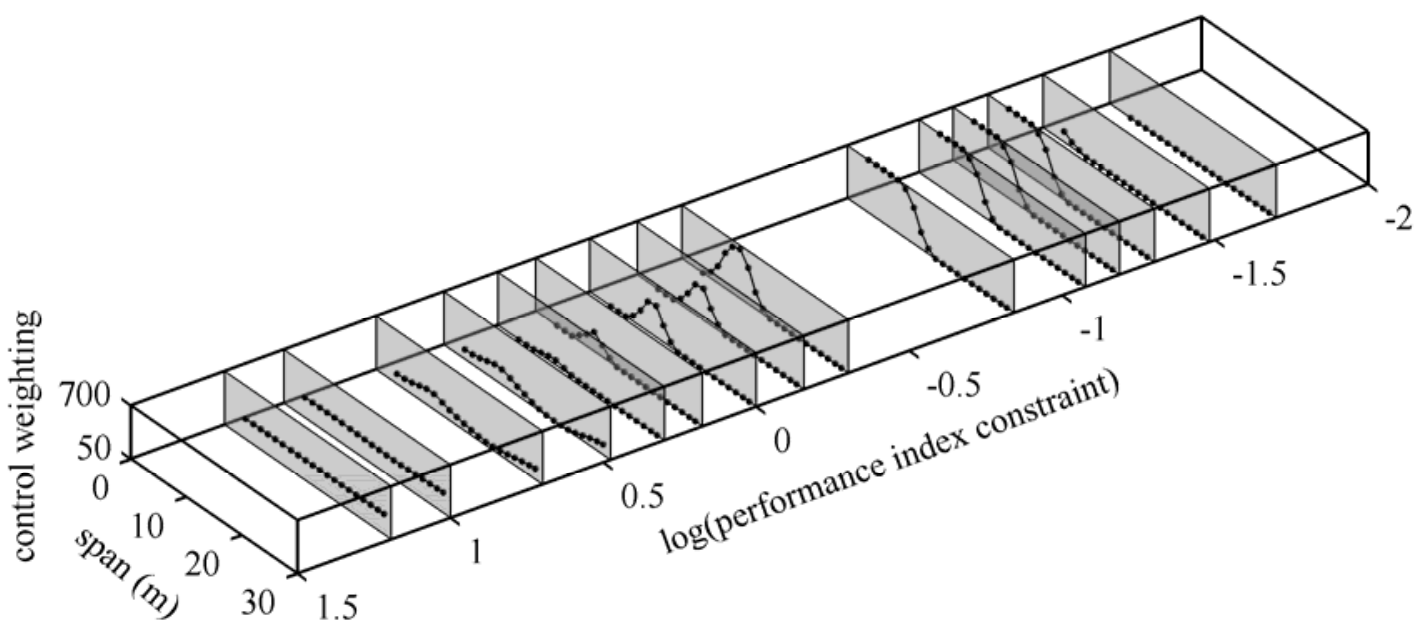

Figure 21. Optimal control weighting terms across the trade-off curve in Figure 20.

\section{Conclusions}

A series of aeroelastic optimization problems are solved in this paper, with increasing complexity in terms of constraints and design variables. All exercises are demonstrated on the UCRM wingbox, an un-deflected jig shape of the Common Research Model with conventional ribs, spars, skins, and smeared stiffeners. Panel method aerodynamics are coupled to shell-based finite element models of the built-up structure, and trimmed longitudinal and lateral maneuver loads are computed. Unsteady aeroelastic effects are computed in the time domain via a Roger transformation of the frequency-domain aerodynamics, and standard LQR controllers are utilized for closed loop mechanics. The following optimization problems are solved:

1. Minimize wingbox mass subject to stress and panel buckling constraints spread across three static aeroelastic maneuver loads. Design variables include shell thickness, stiffener thickness, and stiffener height for design patches spread over the ribs, spars, and skins.

2. Identical to (1), but structural design variables augmented with quasi-steady DMCS rotation variables, one per control surface (20) per static aeroelastic load case (3). Augmenting the design space with DMCS variables allows for an aeroelastically-feasible mass reduction of 31.3\%.

3. Identical to (2), but open loop flutter margin constraints included. This is done by ensuring that the real part of each state eigenvalue is below some constraint curve for all equivalent air speeds of interest, up to the 
desired flutter margin. Satisfying a $20 \%$ flutter margin constraint increases the optimal mass by $34.4 \%$, a penalty that can be much higher if the control surfaces are attached to the wingbox with flexible hinge springs.

4. Identical to (3), but closed loop flutter margin and control cost (performance index) constraints included. Structural and DMCS design variables are augmented with control weighting design variables, as well as the equivalent air speed at which the LQR controller is designed. If higher control cost values are allowed, the LQR controller is able to meet the closed loop flutter constraint without the need to stiffen the structure. More stringent cost allowances do force the optimizer to add structural mass, however.

\section{Acknowledgements}

This work is funded by NASA's Advanced Air Transport Technologies program. Thanks to Dr. Joaquim Martins and Dr. Gaetan Kenway of the University of Michigan for providing the uCRM model.

\section{References}

[1] Shirk, M., Hertz, T., Weisshaar, T., “Aeroelastic Tailoring - Theory, Practice, and Promise,” Journal of Aircraft, Vol. 23, No. 1, pp. 6-18, 1986.

[2] Livne, E., “Integrated Aeroservoelastic Optimization: Status and Direction,” Journal of Aircraft, Vol. 36, No. 1, pp. 122145, 1999.

[3] Raveh, D., "Maneuver Load Analysis of Overdetermined Trim Systems,” Journal of Aircraft, Vol. 45, No. 1, pp. 119-129, 2008.

[4] Reich, G., Raveh, D., Zink, P., “Application of Active-Aeroelastic-Wing Technology to a Joined-Wing Sensorcraft,” Journal of Aircraft, Vol. 41, No. 3, pp. 594-602.

[5] Zink, P., Mavris, D., Raveh, D., “Maneuver Trim Optimization Techniques for Active Aeroelastic Wings,” Journal of Aircraft, Vol. 38, No. 6, pp. 1139-1146, 2001.

[6] Layton, J., "Aeroservoelastic Tailoring for Gust Response of a Typical Section Aeroelastic Model,” AIAA Structures, Structural Dynamics, and Materials Conference, New Orleans, LA, April 10-12, 1995.

[7] Haghighat, S., Martins, J., Liu, H., “Aeroservoelastic Design Optimization of a Flexible Wing,” Journal of Aircraft, Vol. 49, No. 2, pp. 432-443, 2012.

[8] Jackson, T., Livne, E., "Integrated Aeroservoelastic Design Optimization of Actively Controlled Strain-Actuated Flight Vehicles,” AIAA Journal. Vol. 52, No. 6, pp. 1105-1123, 2014.

[9] Zeiler, T., Weisshaar, T., “Integrated Aeroservoelastic Tailoring of Lifting Surfaces,” Journal of Aircraft, Vol. 25, No. 1, pp. 76-83, 1988.

[10] Dracopoulos, T., Oz, H., “Integrated Aeroelastic Control Optimization of Laminated Composite Lifting Surfaces,” Journal of Aircraft, Vol. 29, No. 2, pp. 280-288, 1992.

[11] Nam, C., Kim, Y., “Optimal Design of Composite Lifting Surface for Flutter Suppression with Piezoelectric Actuators,” AIAA Journal, Vol. 33, No. 10, pp. 1897-1904, 1995.

[12] Patil, M., Clark, R., “Aeroelastic Control using Multiple Control Surfaces,” AIAA Structures, Structural Dynamics, and Materials Conference, Denver, CO, April 22-25, 2002.

[13] Zhao, Y., "Flutter Suppression of a High Aspect Ratio Wing with Multiple Control Surfaces," Journal of Sound and Vibration, Vol. 324, pp. 490-513, 2009.

[14] Cavagna, L., Ricci, S., Scotti, A., “Active Aeroelastic Control over a Four Control Surface Wing Model,” Aerospace Science and Technology, Vol. 13, No. 7, pp. 374-382, 2009.

[15] Kenway, G., Martins, J., Kennedy, G., “Aerostructural Optimization of the Common Research Model Configuration,” AIAA Aviation Conference, Atlanta, GA, June 16-20, 2014.

[16] Vassberg, J., DeHaan, M., Rivers, S., Wahls, R., "Development of a Common Research Model for Applied CFD Validation Studies,” AIAA Applied Aerodynamics Conference, Honolulu, Hawaii, August 10-13, 2008.

[17] Cook, R., Malkus, D., Plesha, M., Witt, R., Concepts and Applications of Finite Element Analysis, Wiley, New York, 2002.

[18] Katz, J., Plotkin, A., Low-Speed Aerodynamics, Cambridge University Press, Cambridge, UK, 2001.

[19] Appa, K., “Finite-Surface Spline,” Journal of Aircraft, Vol. 26, No. 5, pp. 495-496, 1989.

[20] Kreisselmeier, G., Steinhauser, R., "Systematic Control Design by Optimizing a Vector Performance Index”, International Federation of Active Controls Symposium on Computer-Aided Design of Control Systems, Zurich, Switzerland, 1979.

[21] Herencia, J., Weaver, P., Friswell, M., "Morphing Wing Design via Aeroelastic Tailoring,” AIAA Structures, Structural Dynamics, and Materials Conference, Honolulu, HI, April 23-26, 2007.

[22] Dillinger, J., Klimmek, T., Abdalla, M., Gürdal, Z., "Stiffness Optimization of Composite Wings with Aeroelastic Constraints,” Journal of Aircraft, Vol. 50, No. 4, pp. 1159-1168, 2013.

[23] Sigmund, O., "Morphology-Based Black and White Filters for Topology Optimization,” Structural and Multidisciplinary Optimization, Vol. 33, pp. 401-424, 2007.

[24] Svanberg, K., "A Class of Globally Convergent Optimization Methods Based on Conservative Convex Separable Approximations,” SIAM Journal of Optimization, Vol. 12, pp. 555-573, 2002. 
[25] Kline, K., “Dynamic Analysis Using a Reduced Basis of Exact Modes and Ritz Vectors,” AIAA Journal, Vol. 24, No. 12, pp. 2022-2029, 1986.

[26] Roger, K., “Airplane Math Modeling Methods for Active Control Design,” AGARD CP-228, pp. 4-11, 1977.

[27] Ringertz, U., “On Structural Optimization with Aeroelasticity Constraints,” Structural Optimization, Vol. 8, pp. 16-23, 1994.

[28] Bae, J., Yang, S., Lee, I., "Linear and Nonlinear Aeroelastic Analysis of Fighter-Type Wing with Control Surface,” Journal of Aircraft, Vol. 39, No. 4, pp. 697-708.

[29] Zhang, Z., Yang, S., Chen, P., "Linearized Euler Solver for Rapid Frequency-Domain Aeroelastic Analysis,” Journal of Aircraft, Vol. 49, No. 3, pp. 922-932, 2012.

[30] Wakayama, S., Kroo, I., “Subsonic Wing Planform Design Using Multidisciplinary Optimization,” Journal of Aircraft, Vol. 32, No. 4, pp. 746-753, 1995.

[31] Stevens, B., Lewis, F., Aircraft Control and Simulation, John Wiley and Sons, Hoboken, NJ, 2003.

[32] Barker, J., Balas, G., "Comparing Linear Parameter-Varying Gain-Scheduled Control Techniques for Active Flutter Suppression,” Journal of Guidance, Control, and Dynamics, Vol. 23, No. 5, pp. 948-955, 2000.

[33] Gilbert, M., “An Analytical Sensitivity Method for use in Aeroservoelastic Aircraft Design,” NASA TM 101583, 1989.

[34] Moulin, B., "Modeling of Aeroservoelastic Systems with Structural and Aerodynamic Variations,” AIAA Journal, Vol. 43, No. 12, pp. 2503-2513, 2005. 